\title{
Leadership and prestige, mothering, sexual selection, and encephalization: The computational services model
}

\author{
Edward Hagen and Zachary Garfield
}

May 26, 2019

\begin{abstract}
Long before the term Machiavellian Intelligence was coined, James Neel, a prominent geneticist, was pondering the role of 'princes' in the evolution of exceptional human intelligence. Neel discovered that in small-scale societies resembling those in which humans evolved, leaders had more wives and more children than other men. If this pattern, which is now well-established in the ethnographic record, characterized ancestral human societies, whatever traits predisposed men to become leaders would have experienced strong sexual selection. Neel proposed that the key trait was intelligence. Sexual selection on intelligent leaders therefore helped explain human encephalization.

Many subsequent theories have attempted to explain why knowledgeable, skilled, and intelligent individuals are chosen as leaders or as mates. None, however, has adequately explained why they are chosen as both. We aim to fill this gap by operationalizing leaders as individuals who regularly make decisions that benefit most members of the group. Because human nuclear families comprise two unrelated individuals who cooperate for twenty years or more to raise their joint offspring, and because families are nested within subsistence groups, which, in turn, are nested within larger security and political groups, good decision-making skills provided large benefits to mates and families, as well as to members of one's subsistence group or larger security and political groups. We further argue that decision-making that benefits others as well as oneself can be especially computationally complex, and therefore that sexual selection and biological market forces favoring these skills favored increased brain size. Finally, because parents must make decisions for their cognitively immature offspring, decision-making that benefits others and other leadership abilities might have initially undergone strong selection in mothers, who provide most of the childcare in natural fertility populations.

Decision-making that benefits others is one valuable example of what we term a computational service. Other important examples include threat and opportunity detection, gossip and information sharing, cultural transmission, story telling, medicinal knowledge, and advice and counsel. Providing computational services in exchange for a variety of benefits would have helped subsidize a large, energetically expensive brain. Individuals who provided particularly valuable services gained prestige, i.e., additional benefits from fellow group members.
\end{abstract}

\section{Introduction}

Humans, like many other social species, exhibit social hierarchies in which top-ranked individuals typically have preferential access to resources and mates. In humans and other animals, these hierarchies are often based on physical formidability and/or the formidability of within-group coalitions. A compelling theoretical explanation for the formation of such dominance hierarchies is that they reduce the cost of fighting over resources (Maynard Smith \& Parker, 1976; Drews, 1993). Human social hierarchies, however, are also based on asymmetries in knowledge and skill. Knowledgeable and skilled individuals acquire prestige, are deferred to by others, have increased mating success, and often ascend to leadership positions. Although many theories have been put forward for the evolution of knowledge- and skill-based social hierarchies, we will argue here that each has empirical and/or theoretical deficiencies.

James Neel, a major figure in twentieth century genetics, was perhaps the first to recognize the evolutionary implications of knowledge-based social rank. He observed that in small-scale societies leaders are often polygynous and have more children than other men (Neel et al., 1964; Neel \& Salzano, 1967; Neel, 1980). Neel reasoned that if this pattern characterized most human societies during our evolution, there 

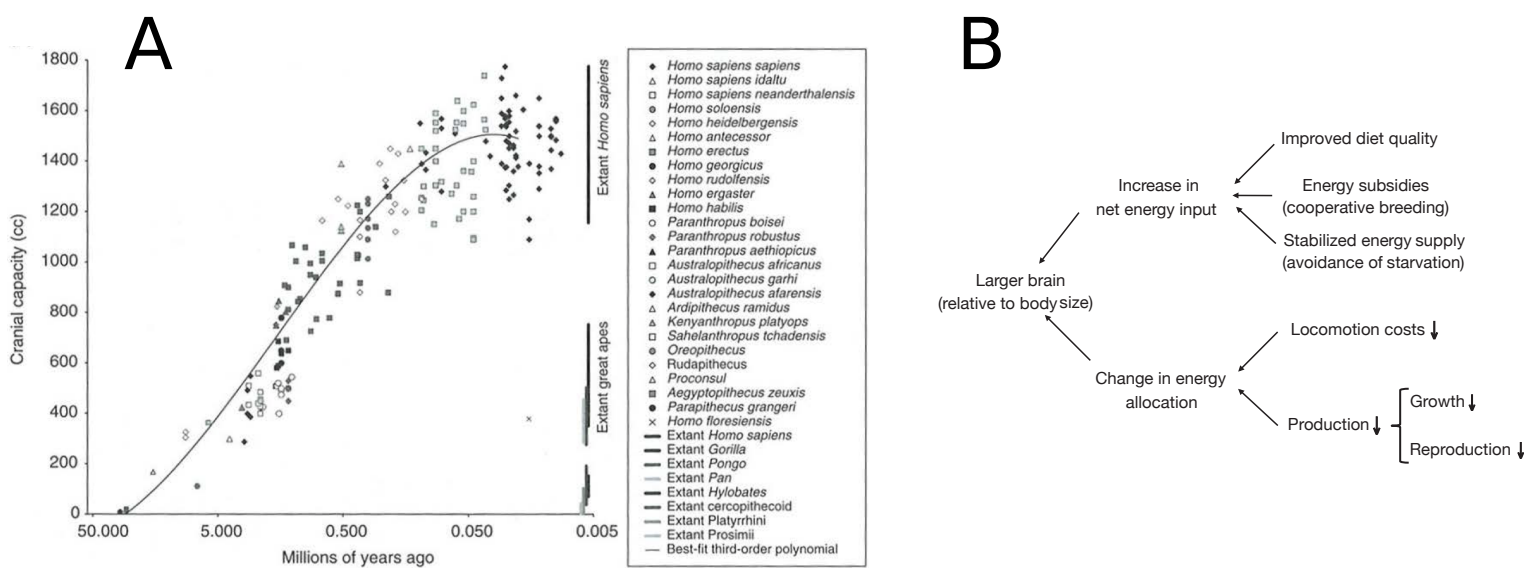

Figure 1: A. Evolution of primate cranial capacity. Each point is one fossil specimen. Figure A from Schoenemann (2013). B. The expensive-brain framework proposes complementary pathways for an adaptive increase in relative brain size. First, brains can get larger when energy inputs are stabilized on a higher level (higher total metabolic turnover) through an increase in mean dietary quality (for example, more animal fat and protein in early Homo), energy subsidies from other individuals (for example, cooperative breeding, allomaternal care) or by reducing fluctuations in energy inputs (for example, cognitive solutions, including culture). Second, at constant total energy intake, energy allocation to other functions may be reduced, such as locomotion (for example, efficient bipedalism) or production (for example, slower life history pace). Figure B and caption from Navarrete et al. (2011).

would have been strong sexual selection for the trait(s) that predisposed men to ascend in social rank and become community leaders. Many later authors have argued that this trait is status striving, i.e., that the reproductive benefits of prestige and leadership roles would select for a strong motive in men to achieve high status (e.g., Irons, 1998; Geary \& Flinn, 2001; Gurven et al., 2009; von Rueden \& Jaeggi, 2016; Van Vugt \& Tybur, 2014).

Neel had something quite different in mind. Political leaders in small-scale societies are chosen by other men. What traits would men value in other men? Leaders are often skilled hunters, warriors, and orators, and masters of tribal lore. Neel therefore argued that although physical strength is an asset in campaigns for leadership in small-scale societies, mental agility is even more critical. Neel proposed that there was sexual and social selection, not on status striving per se, but on cognitive abilities generally (Neel \& Salzano, 1967; Neel, 1980). Because sexual selection often results in exaggerated traits, this could explain the dramatic increase in human cranial capacity in genus Homo (Figure 1A).

The human brain is energetically expensive to run, consuming $20-25 \%$ of basal metabolic rate. Human encephalization, which Kaplan et al. (2003) refer to as investment in neural capital, required some combination of increased energy intake and/or reduced energy allocation to other tissues relative to hominin and ape ancestors (e.g., Isler Karin \& van Schaik Carel P, 2006; Navarrete et al., 2011, and references therein). See Figure 1B. A longstanding goal in evolutionary anthropology is to determine the fitness benefit(s) of investing increased energy in the brain vs. other tissues and activities. Two broad categories of possible answers, based largely on cross-species comparisons, are an increased ability to solve either social problems, often termed Machiavellian Intelligence (e.g., Byrne \& Whiten, 1988; Whiten \& Byrne, 1997; Humphrey, 1976) or ecological problems (e.g., Milton, 1988); social learning and culture are often added into the mix as solutions to both types of problems (for recent treatments of these hypotheses, see, e.g., Rosati, 2017; Gonzalez-Forero M \& Gardner A, 2018; Ashton et al., 2018; Muthukrishna et al., 2018; DeCasien et al., 2017; Street et al., 2017; Powell et al., 2017). Neel and colleagues were therefore among the first to propose an evolutionary theory of encephalization that combined elements of the social intelligence hypothesis, the ecological intelligence hypothesis, and the cultural brain hypothesis.

Our paper is organized as follows. First, we summarize the evidence that, over human evolution, high status men had more mates and offspring than other men. We then summarize the evidence for sexual body 
dimorphism that might be a consequence of male-male physical competition for mates. This evidence suggests that sexual selection operated on human evolution, and that physical dominance played an important role in human status hierarchies. Dominance theories are then contrasted with theories, like Neel's, that emphasize the role of knowledge and intelligence in status and leadership. We argue that although each of these theories has contributed to our understanding of the evolution of human prestige and leadership, none is completely satisfactory. Neel, for example, did not explain how general cognitive abilities benefited other men, nor why leaders would be favored as mates. Building on the work of many others, we fill in some of the theoretical gaps in existing theories with a detailed explanation of our computational services model, which we briefly sketched in Garfield et al. (2018) and Garfield et al. (2019). A surprising consequence of our model is that selection for the cognitive abilities underlying leadership might have initially occurred in females.

\section{Sexual selection in human evolution}

Darwin was the first to propose that differential success in the competition for mates, which he termed sexual selection, explained the evolution of otherwise puzzling traits, such as elaborate ornaments and weaponry (Darwin, 1871). There could be sexual selection for traits that provide an advantage in competition among members of the same sex for members of the opposite sex (e.g., weapons in male-male competition), as well for traits that increase the likelihood of being chosen as a mate (e.g., ornaments in female choice). A further distinction involves pre-copulatory factors (e.g., mate choice) vs. post-copulatory factors (e.g., sperm competition). Since Darwin, an enormous quantity of empirical evidence has accumulated supporting the role of sexual selection in the evolution of a very wide range of species (Andersson, 1994; Jones \& Ratterman, 2009), including humans (Miller, 1998; Puts, 2016).

\subsection{Male reproductive skew}

There is substantial evidence that leaders and other high status men have more mates and offspring than other men. Such biased reproduction, also referred to as reproductive skew, is observed in many non-human species (Vehrencamp, 1983; Kokko, 2003; Shen \& Reeve, 2010). In non-human primates, for example, the association between male status and reproductive success (RS) is $r=0.80$ (Cowlishaw \& Dunbar, 1991). von Rueden \& Jaeggi (2016) conducted a meta-analysis of the association between male status and various indices of reproductive success (RS) in 33 non-industrial societies that included hunter-gatherers, pastoralists, and agriculturalists. Although the mean effect size, $r=0.19$, was smaller than the effect seen in non-human primates, it was significantly greater than 0 for most indices of RS, and did not vary among societies of different subsistence types. See Figure 2A.

Genetic evidence indicates that ancestral human societies also exhibited male reproductive skew. By comparing variation in mtDNA (inherited from mothers only) to non-recombining Y chromosomal regions (inherited by sons from fathers only) in a large multi-regional sample of genomes, Lippold et al. (2014) and Karmin et al. (2015) both conclude that, pre-dating the migration of modern humans from Africa, female effective population size was consistently larger than that of males, i.e., relatively fewer males reproduced. This could indicate either a long evolutionary history of polygyny, sex-specific migration, and/or matrilineality (Oliveira et al., 2018).

Tentatively interpreting the results from Karmin et al. (2015) as evidence of male reproductive skew (Figure 2B), there were approximately 3 reproducing females for every reproducing male from 140-30 thousand years ago (KYA), with some fluctuation during the expansion of out Africa c. 80-50 KYA. This $100 \mathrm{KYA}$ time span might have been sufficient for sexual selection to have acted on the evolution of traits underlying leadership, prestige, and mating, especially if the pattern seen here extended even further into the past. ${ }^{1}$

\footnotetext{
${ }^{1}$ The dramatic increase in this ratio starting after the glacial maximum c. $20 \mathrm{KYA}$, peaking at $>16$ in the early-to-mid Holocene, has been attributed to a combination of a transition to patrilineal social organization coupled with intensive warfare that would have killed many men in some patrilineages, leading to extinction of their Y-chromosome lineages, and hence low $\mathrm{Nm}$. Women, on the other hand, would not have been killed but instead would have joined the victors' patrilineages (Zeng et al., 2018). These factors, combined with other sociocultural factors such as the emergence and expansion of inequality, concentrations of power and wealth, and social prestige have likely contributed to increased variance in reproduction among human males in the last 10,000 years (Heyer et al., 2012; Karmin et al., 2015; Webster \& Wilson Sayres, 2016). The potential impact of sexual selection over this much shorter time period, however, is less clear.
} 
A

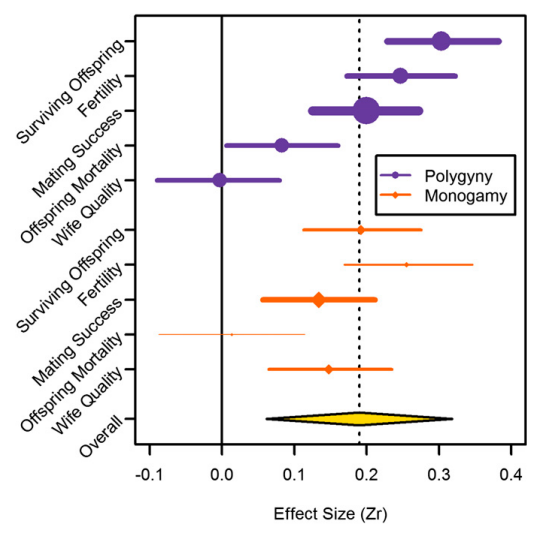

B

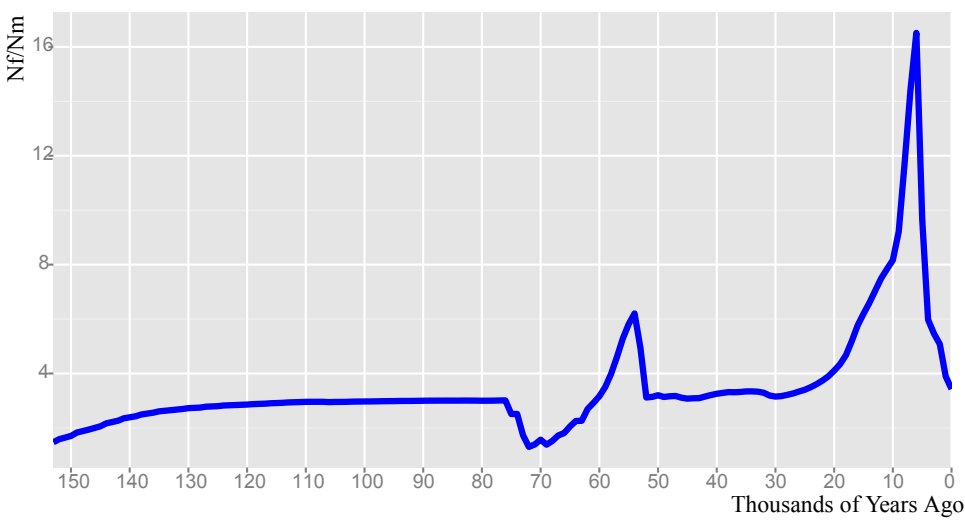

Figure 2: A. Variation in the weighted effect of male status on RS, as a function of RS measure and marriage system (polygyny vs. monogamy) based on averaged coefficients. Overall effect size based on intercept-only model. Before metaanalysis, all effect sizes were coded such that positive signs indicate positive contributions to RS (e.g., a negative effect of status on offspring mortality was coded as positive). Point size and line width are proportional to the number of results contributing to each weighted effect size. Figure A and caption from von Rueden \& Jaeggi (2016). B. The temporal dynamics of the ratio of female (Nf) and male (Nm) effective population size in the last 140KY. The ratios of the global accumulative Ne estimates of mtDNA (Nf) and Y chromosome (Nm) are plotted against the time (in thousands of years) back from the present (0). The BSPs estimates of Ne were obtained in BEAST using a piecewise-linear coalescence model. Figure B and caption from Karmin et al. (2015).

It is not clear whether male reproductive skew in contemporary small-scale societies or in ancestral populations is primarily a result of male-male competition, female choice, some combination of the two, or other processes. Moreover, evidence that, over human evolution, women were mating with a subset of adult men does not, in and of itself, prove that male traits were shaped by sexual selection.

\subsection{Constraints on the evolution of sexual dimorphism}

The cross-cultural evidence for reproductive skew among high status men, and the evidence of skew in ancestral populations, implies that there has been a selection pressure for male traits that would increase status. But there are constraints on the evolution of such traits. For example, humans have 23 pairs of chromosomes, 22 of which are autosomes that are present in both sexes, and one of which comprises the sex chromosomes. Like most mammals, humans have an XY sex-determination system: individuals with two $\mathrm{X}$-chromosomes develop into females and individuals with an $\mathrm{X}$ and an $\mathrm{Y}$ chromosome develop into males. The Y-chromosome is therefore the only one that is exclusive to one sex, and it is quite small, with fewer than 100 genes, only one of which, SRY, determines male sex. Hence, SRY is simply a switch. Most of the sex-specific functionality of males and females, such as testes vs. ovaries, is encoded in the autosomes. In fact, SRY triggers the differentiation of the testes by acting on an enhancer of SOX9, an autosomal gene (Jobling \& Tyler-Smith, 2017).

A key implication is that a new allele that appears on an autosome or the X-chromosome would often be expressed in both sexes. Intralocus sexual conflict arises when the allele codes for a trait that provides a fitness benefit to one sex but imposes a fitness cost on the other. Intralocus conflict would then select for modifier alleles that suppress expression of the trait in the disadvantaged sex, resulting in sexual dimorphism (Rice, 1984; Chapman et al., 2003; but see Queller \& Strassmann, 2018). Yet modifier alleles might not completely suppress the trait - male nipples are a classic example. Thus, arguments for sexual selection on a trait in one sex must always consider the costs and benefits of the trait in the other sex, an issue to which we shall return. 


\subsection{Sexual dimorphism in human body size, strength, and aggression}

Male-male physical competition for mates often selects for sexual body dimorphism. Most primates, including humans, are sexually dimorphic to some degree (Kappeler \& Van Schaik, 2004). The strong sexual body dimorphism among our australopithecine ancestors suggests strong sexual selection, male-male competition, polygyny and substantial levels of reproductive skew, although other explanations are possible (for discussion, see Plavcan, 2012b). Early Homo might also have been markedly sexually dimorphic, although the evidence is far from clear (Plavcan, 2012a).

In modern humans, on the other hand, body dimorphism is modest - men weigh about $15 \%$ more than women - but this is more than gibbons and a number of strictly monogamous and polyandrous primate species (Plavcan, 2012b). Further, human upper body strength is highly sexually dimorphic: in over $90 \%$ of chance encounters between an adult man and woman, the man would have higher upper body strength (Pheasant, 1983; Plavcan, 2012b), a difference that emerges with sexual maturation. See Figure 3. This sex difference in upper body strength, along with a large male bias in physically aggressive behavior, indicates that physical male-male competition for mates, i.e., intrasexual selection, was important during the evolution of modern humans (e.g., Archer, 2004; Archer \& Thanzami, 2009; Puts, 2016). Sexually dimorphic strength could also indicate a sexual division of labor in which men relied on upper body strength to, e.g., hunt big game whereas women hunted smaller game and/or foraged for plant foods (e.g., Gurven et al., 2009), or male defense against predators (DeVore \& Washburn, 1963; Willems \& van Schaik, 2017), hypotheses that are not mutually exclusive.

\section{Dominance, prestige and leadership in non-human animals}

Physically fighting over scarce resources is costly. To avoid paying these costs, many animal species form relatively linear hierarchies based on physical formidability. With an established rank determining access to resources, individuals avoid the cost of a fight (Maynard Smith \& Parker, 1976; Drews, 1993). In many primate species, dominance hierarchies are solidified, or challenged, via alliances with other group members. Thus, in species with complex social relationships, dominance hierarchies typically involve both agonism and prosociality (Harcourt \& De Waal, 1992).

Leaders are defined as individuals that have a disproportionate influence over group decisions (Smith et al., 2016; Kantner, 2010; Van Vugt, 2006; von Rueden et al., 2014). Leadership is distinct from the closely related concepts of dominance, high rank, and social status, which involve increased access to contested resources and/or greater deference from others but not necessarily influence over group behavior. In non-human animals, the distinction between leadership and dominance or social rank is not always clear. Often leadership and dominance are synonymous, as in the case of mountain gorillas (Gorilla beringei beringei) (Fossey, 1972), but in other species and contexts dominant individuals are not necessarily leaders, and leadership can be distributed across individuals, as is the case among migrating groups of white-faced capuchins (Cebus capucinus) (Leca et al., 2003).

In some cases, animal leadership is based on informational asymmetries, which, in analogy with the human case, we will refer to as 'prestige'. Chimpanzee leaders can effectively communicate information on the location, quality, and quantity of resources to the group (Menzel, 1971), and chimpanzees also appear to defer towards, and preferentially learn from experienced individuals (Horner et al., 2010; Kendal et al., 2015). Couzin et al. (2005) model the emergence of leadership among nonhuman animal groups and demonstrate that large groups of individuals can achieve consensus in direction of movement relying exclusively on the movements of relatively few informed leaders. Social learning biased towards older, experienced individuals plays a role in some avian migration (Mueller et al., 2013; Berdahl et al., 2018). Among elephant species (e.g., Loxodonta africana), older matriarchs with special knowledge and experience are the primary decision makers in the group (Payne, 2003) and among killer whales (Orcinus orca) post-reproductive females lead foraging movement, especially during times of limited food resources (Brent et al., 2015). In many species cultural learning and informational asymmetries influence hierarchy formation, beyond the relatively simple heuristic inter-individual cognitive mechanisms (Sapolsky, 2005; Chapais, 2015; Couzin \& Krause, 2003). 


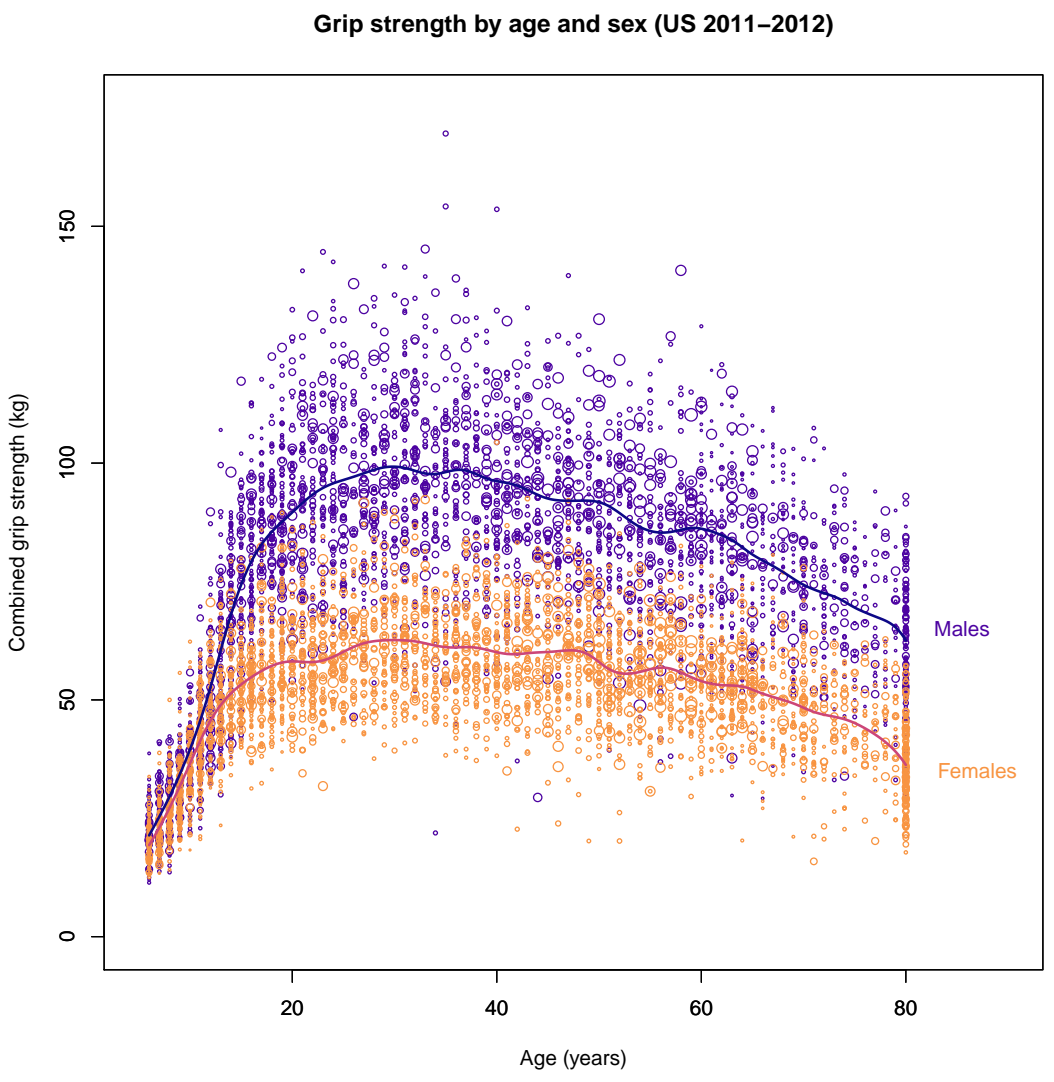

Figure 3: Combined grip strength by age and sex. Combined grip strength is the sum of the largest isometric grip strength readings from each hand, measured using a handgrip dynamometer. Grip strength is an index of upper body strength. Each point is one person. Sample size $=7064$. Data are from the US National Health and Nutrition Examination Survey (NHANES) 2011-2012. NHANES is a representative sample of the US non-institutionalized civilian resident population of the United States. It utilizes a complex, multistage, probability sampling design. The sizes of the symbols represent the sampling weights. 


\section{Dominance in human societies}

Humans, like many other social species, form social hierarchies that regulate access to resources and involve both agonism and prosociality (e.g., Hawley, 1999). Tiger \& Fox (1971) argued that human societies could be best understood in light of our primate heritage, specifically a heritage involving male dominance hierarchies in which high ranking males had privileged access to fertile females and resources (see also Tiger, 1970). On their view, a human society's political system "is a breeding system" (Tiger \& Fox, 1971, p. 25). Leaders are dominant, and typically older, males, who command "attention," a construct they borrow from the primatologist Chance (1967), and who control the distribution of resources in the group. They also acknowledge the role of prestige: "The successful hunter...has to convert surplus [food] into prestige. In return he will be deferred to and also will be allowed to accumulate the primeval liquid asset - women" (Tiger \& Fox, 1971, p. 124).

Human male dominance hierarchies based on aggression and fear would appear to be homologous to nonhuman primate status hierarchies (Tiger \& Fox, 1997; Barkow, 1989; Henrich \& Gil-White, 2001; Chapais, 2015). Such dominance hierarchies have solid theoretical foundations in evolutionary game theory (Maynard Smith \& Parker, 1976; Drews, 1993), are associated with reproductive skew, and are well-supported in the ethnographic record (Garfield et al., 2019, 2018).

\section{$5 \quad$ Prestige and leadership in human societies}

As in many animal species, human status also appears to depend on asymmetries in information, not just physical formidability. Many scholars have discussed the importance of intelligence and knowledge in leadership (e.g., Judge et al., 2004; Roscoe, 2007; Van Vugt \& Kurzban, 2007; Wilson et al., 1996; Connelly et al., 2000; Cavazotte et al., 2012; Henrich et al., 2015), and there is an association between social dominance and rapid decision-making (da Cruz et al., 2018). Garfield et al. (2019) analyzed 1212 ethnographic records on leadership from 58 diverse cultures in the Human Relations Area Files (HRAF). Each record was coded on numerous variables drawn from the theoretical literature on leadership, including those related to dominance and prestige. More than $70 \%$ of cultures characterized leaders as intelligent, knowledgeable, having expertise, and providing counsel. We term leaders in this mold as prestige-style leaders.

Prestige-style leadership in the small-scale, egalitarian societies that are thought to be similar to those in which humans evolved is often ephemeral, context specific, and primarily dependent on mutually beneficial outcomes for leaders and followers (Fried, 1967; Price \& Van Vugt, 2014). Leaders do not have any formal authority, but instead typically gain influence through respect and deference for expertise in culturally valued skills, such as subsistence efforts, oratory abilities, shamanism, and through success in warfare or inter-group conflict (Henrich et al., 2015). Critically, influence is generally maintained only to the degree the group permits (Boehm, 1993; Fried, 1967; Service, 1964; Woodburn, 1982). Therefore, the qualities of prestigestyle leaders and the functions they serve are often, but not always, prosocial in nature (Henrich et al., 2015; Macfarlan et al., 2012).

Leaders are commonly responsible for resolving within-group conflicts across many small-scale societies (Glowacki \& von Rueden, 2015). Mechanisms for conflict resolution among egalitarian hunter-gatherers have been debated, however, with some researchers suggesting egalitarian hunter-gatherers generally lack effective cultural institutions and leadership structures to mediate significant conflicts with clashing parties most often choosing to "vote with their feet" and leave the group (Knauft et al., 1991; Wiessner, 2016). Spiritual beliefs and fear of supernatural punishment are also implicated in promoting social cohesion in the absence of more formal mediation (Basedow, 1925; Lewis, 2008). Other scholars have suggested concerted processes of conflict resolution are ubiquitous among egalitarian societies and highlight the senior role of kin group members (Hames, 2015) and the cost of migrations even among highly nomadic populations (Boehm, 1999; Knauft et al., 1991).

From the ethnography of prestige and leadership in small-scale societies, it is clear that leaders and other high status individuals typically do not have formal authority and there is no requirement that anyone listen to them or follow their decisions. Instead, their views and opinions carry weight. This strongly implies that, through past experience or independent evaluation, their social partners expect to benefit from these views and opinions. 


\subsection{Sexual selection theories of leadership and prestige}

Neel was perhaps the first to put forward an evolutionary theory of human leadership based on knowledge, skill, and intelligence (Neel et al., 1964; Neel \& Salzano, 1967; Neel, 1980). Neel \& Salzano (1967) write:

Among the Xavante, the number of wives is a measure of social status (Maybury-Lewis, 1967). This was true for other Indian groups (e.g., Hallowell, 1938; Levi-Strauss, 1944; Dunning, 1959) and may have been a feature of the breeding structure of many or most primitive groups (e.g., Eskimos: Jenness, 1922; Dunning, 1962). This social status is usually earned; hunting-gatheringincipient agriculture Indian societies were relatively democratic and equal in opportunity (cf. Hallowell, 1963). In general, the leaders will be accomplished orators, good hunters and warriors, well versed in the tribal lore. In these small communities, one's performance under widely varying conditions is well known; it seems reasonable to postulate that the leaders will tend to have intellects and physiques which in that culture are superior.

Neel and Salzano then perform a back-of-the-envelope calculation that shows that if men become leaders due to their intelligence, their reproductive success would result in strong selection on intelligence. Neel (1980) elaborated on this hypothesis, noting that "While physical strength is an asset, I suggest that mental agility is even more important: [the headman] will not be stupid" (p. 283).

Barkow independently argued that human status is better described as an exaptation of dominance that is based on knowledge and skill, which he refers to as prestige. He might also have answered one of the questions raised by Neel's theory: why leaders are attractive as mates. Prestige, according to Barkow, is based much more on female choice than on physical male-male competition. Women preferred to mate with men who could provide the resources necessary to support years of investment in highly dependent offspring. Obtaining these resources required culturally acquired knowledge and skills. Hence, human males evolved to increase their attractiveness to potential mates by competing with each other to acquire and display the skills and knowledge valued by females, i.e., by gaining prestige, rather than gaining mates exclusively via physical competition with other men (Barkow et al., 1975; Barkow, 1989).

\subsubsection{Sex differences in cognition, and the lack thereof}

Neel's and Barkow's theories both posit strong sexual selection on cognitive abilities in men. In support, there are many sex differences in psychology that are likely consequences of sexual selection. In diverse crosscultural samples, for example, there are large female biases in preferences for mates that are, e.g., older, high status, and ambitious (Conroy-Beam et al., 2015), and there is also a clear male bias in preference for multiple sex partners (Schmitt, 2003, 2005). These sex differences in preferences appear to result in sex differences in behavior (Buss \& Schmitt, 2019), and they align very well with Barkow's theory.

There are also some robust sex differences in spatial cognition, e.g., a large male bias in mental rotation (Levine et al., 2016) and a smaller female bias in object identity and location memory (Voyer et al., 2007), which could be due to the putatively greater range size of ancestral men compared to women (Gaulin \& FitzGerald, 1986; Miner et al., 2014), and/or male hunting vs. female gathering (Silverman \& Eals, 1992). These differences might be linked to achieving leadership roles (e.g., as a hunt leader) and female choice (e.g., an increased ability to provide resources to potential mates), but they could also simply reflect specializations to sex-specific economic niches. Sex differences in mating and spatial cognition demonstrate that some aspects of human psychology are plausibly a consequence of sexual selection (for review and discussion, see Ball et al., 2014). They do not, however, provide evidence that there was sexual selection on overall cognitive capacity.

Sex differences in brain size are more promising evidence of sexual selection on cognitive capacity. Across a wide range of mammalian and bird species, absolute brain volume is the best predictor of cognitive performance (MacLean et al., 2014). Within humans, a meta-analysis of sex differences in absolute brain volume found that, on average, males have absolutely larger intracranial volume (12\%), total brain volume $(11 \%)$, cerebrum $(10 \%)$, grey matter $(9 \%)$, white matter $(13 \%)$, cerebrospinal fluid $(11.5 \%)$, and cerebellum (9\%) (Ruigrok et al., 2014). Moreover, there is a robust (albeit small) association of brain volume and IQ (Pietschnig et al., 2015; Nave et al., 2019; Cox et al., 2019), which would seem to indicate that there should be a clear male advantage in IQ. Contrary to this hypothesis, there is not (Pietschnig et al., 2015; Nave et al., 
2019). Nor is there a male advantage in scholastic achievement; if anything, there is a female advantage (Voyer \& Voyer, 2014).

In contrast to the dramatic male bias in upper body strength and physical aggression, which supports an evolutionary history of intrasexual selection based on male-male physical competition for mates, the lack of any male bias in IQ or scholastic achievement undermines Neel's sexual selection argument for encephalization in Homo. Perhaps alleles for increased cognitive capacity were sexually selected in males but had little-to-no cost when expressed in females, i.e., there was little intralocus sexual conflict. Women would then have large brains as a byproduct of sexual selection on men. The brain is extremely energetically expensive, however, representing about $2 \%$ of body mass yet consuming up to $25 \%$ of total energy intake (Herculano-Houzel, 2012), which suggests that if there were no fitness benefit, there should have been strong selection against larger brain sizes in females. On current evidence, then, any theory of encephalization in Homo must identify fitness benefits of larger brains for both males and females.

\subsection{Cultural transmission theories of prestige and leadership}

Henrich \& Gil-White (2001) critiqued the Barkow (1980) account of human prestige: why should men defer to other men who are better able to provide resources to women? Henrich \& Gil-White (2001), drawing on geneculture coevolutionary theory (Boyd \& Richerson, 1985; Cavalli-Sforza et al., 1982; Cavalli-Sforza \& Feldman, 1981), instead argue that prestige is grounded in social learning. Due to differential skill levels in culturally learned behaviors, less-skilled individuals would benefit by learning from the most-skilled individuals. By showing deference to those with greater knowledge and skills, the less knowledgeable and skilled can gain access to them so as to acquire their knowledge and skills. Once common, such patterns of deference could then be utilized by new learners to decide from whom to learn, i.e., copy the most 'popular' or prestigious members of the group (Henrich \& Gil-White, 2001). Thus, like the theories of Neel and Barkow, this theory has strong parallels with the information-based theories of animal leadership (c.f., Chapais, 2015).

Henrich et al. (2015) extend the foregoing model by showing how group members can become more cooperative by copying cooperative leaders, which can then drive natural selection on leaders to be even more cooperative. Their Big Man Mechanism suggests cooperation is often rooted in prestige-based leadership, prestige-biased learning, and positive assortment of leaders and followers. In the Henrich et al. (2015) models, cooperation can emerge from emulation biases, and can be maintained in the absence of punitive sanctions if followers are preferentially modeling their behavior after prosocial leaders. Leaders whose influence stems from information-based prestige can expand their influence via other strategies, including dominance and non-informational prestige (Henrich et al., 2015).

Henrich and colleagues also argue that the rapid encephalization among humans was driven by pressures for social learning and cumulative culture. The Cultural Brain Hypothesis $(\mathrm{CBH})$ predicts larger brains were selected for due to their capacity for storage and management of increasingly complex and locally adaptive cultural information across a wide range of behavioral and social domains (Richerson \& Boyd, 2000, 2008; Muthukrishna et al., 2018).

Many studies in Western populations provide evidence that learners preferentially copy, and direct attention to, prestigious individuals and those that are high in the social hierarchy (Henrich \& Henrich, 2007; Richerson \& Henrich, 2012; Cheng et al., 2010, 2013; Foulsham et al., 2010; Maner et al., 2008). The prestigebiased learning model is also consistent with the lack of a sex difference in IQ or scholarly achievement, as women can equally serve as prestigious sources of knowledge (Henrich \& Henrich, 2010; Placek et al., 2017).

The prestige-biased learning model has several theoretical and empirical problems, however. First, it does not directly account for the increased mating success of prestigious men, nor the pronounced malebias in leadership. Second, as followers acquire the knowledge and skills of prestigious leaders, the supply of knowledgeable individuals increases and the demand for this knowledge decreases. Hence, the 'price' to obtain this knowledge - levels of deference - should decrease, i.e., the prestigious would lose their prestige. To maintain prestige, individuals would have to acquire valuable knowledge, e.g., via individual learning, at a rate that equals or exceeds its dissipation via social transmission. Third, other than young individuals copying older individuals, extensive surveys of the ethnographic record have failed to find many examples of prestige-biased emulation (Garfield et al., 2019, 2016), although among the Chabu, Garfield \& Hagen (2019) did find that emulation was related to prestige (but much less so to leadership). Thus, although cultural transmission was probably aided by juveniles and young adults with less knowledge and 
skills deferring to older adults with more knowledge and skills so as to learn from them (vertical and oblique transmission), cultural transmission seems less able to explain the deference of fully mature adults to other adults. Jiménez \& Mesoudi (2019) review existing evidence and outstanding questions relevant to the Henrich \& Gil-White (2001) model of prestige-biased social learning. Overall, they find mixed support and conclude positive relationships between prestige and knowledge are likely dependent upon several factors including the stability of the local social and ecological environment, the availability and viability of other learning biases, and the social complexity (i.e. degree of role specialization) of the population.

\subsection{Collective action theories of leadership}

Another major approach to the evolution of leadership emphasizes the role of leaders in collective actions. Humans, as a species, are reliant on high levels of coordination and cooperation among groups of individuals who are often either distant relatives, or non-relatives. As group size increases and cooperative tasks become more complex, the likelihood that collective actions can fail due to, e.g., free-riding or conflicts of interest among participants increases (Axelrod \& Hamilton, 1981; Olson, 1965). Many researchers have proposed that leadership might have evolved, at least in part, to solve such collective action problems by monitoring individual behavior, sanctioning free-riders, rewarding contributors, and solving coordination problems (Hooper et al., 2010; Tooby et al., 2006; Glowacki \& von Rueden, 2015; Van Vugt \& Kurzban, 2007; Price \& Van Vugt, 2014; Ruttan \& Borgerhoff Mulder M, 1999; Gavrilets \& Fortunato, 2014). The main idea is that leaders will assume the costs of leadership to the extent they are compensated by followers, group returns, or positive reputations that attract future aid and mating opportunities (e.g., Glowacki \& Wrangham, 2013; Hooper et al., 2010; Smith \& Choi, 2007). Some theories particularly emphasize the important role of leaders in aggressive collective actions against other groups - warfare (Glowacki et al., 2017; Lopez, 2016; Johnson, 2015; Gavrilets \& Fortunato, 2014).

The emergence of leaders in work groups is a paradigmatic example of leaders' roles in collective actions. "Chiefs-for-a-day" are individuals who take on the role of being the leader and ensure a cooperative task is completed efficiently. Typically, though, the social influence of chiefs-for-a-day does not extend to other social or economic domains (Macfarlan et al., 2012).

\subsection{The service-for-prestige model}

Price \& Van Vugt $(2014,2015)$ generalize the foregoing models. They propose that leaders, at some fitness cost to themselves, provide valuable services to followers, such as knowledge, expertise, or organizational skills. In return, followers, at some fitness cost to themselves, provide "prestige" to leaders, which translates into, e.g., resources or mates. This model therefore proposes a form of reciprocal altruism between leaders and followers. Price \& Van Vugt (2014) acknowledge that their model faces a 'public goods' problem, in that leader services are a public good, and thus followers have an incentive to free-ride by failing to defer to leaders. They propose that punishment can help solve the free-rider problem. Specifically, followers who do not appropriately respect beneficial leaders will be socially sanctioned by other group members, whereas followers who show contempt for non-beneficial leaders will be rewarded by other group members. The degree to which followers reward or sanction other group members for their followership (or lack thereof) remain open questions, however, ones that Price \& Van Vugt (2014) believe constitute the most important tests of their model.

\subsection{The conundrum of prestige}

Social status and leadership based on skill, knowledge, intelligence, and expertise is a conundrum. In our view, a theory of prestige must explain (1) the importance of skill, expertise and intelligence to the acquisition of prestige, (2) the deference of adult members of the same sex to other prestigious members of the same sex, (3) the increased reproductive success of prestigious men, (4) the influence of prestigious men over group decisions, (5) the generosity of leaders, and (6) the lack of a sex difference in IQ or scholarly achievement. Although each of the theories discussed here provides important insights, none satisfies all these criteria. Barkow (1989) persuasively argued that females would prefer men with culturally acquired skills and expertise because, compared to other men, they could provide the more resources directly to their 
mates. We agree with Henrich \& Gil-White (2001), however, that this does not explain why men should defer to other skilled men, which is better explained by the prestige-biased emulation model. Yet Henrich \& Gil-White (2001) do not explain how prestige results in male reproductive skew, nor why men (but not women) who are emulated for their culturally and individually acquired knowledge also have disproportionate influence over group decisions. Furthermore, to maintain their prestige, individuals would have to acquire new knowledge as their dissipated knowledge loses its value. Finally, systematic cross-cultural analyses of the ethnographic record have not found much evidence for prestige-biased emulation among adults (Garfield et al., 2016, 2019).

We agree with Neel that higher rates of polygyny and reproductive success among leaders, if characteristic of Homo sapiens, would represent tremendous sexual selection for the evolution of traits predisposing to leadership, primarily universal aspects of prestige: intelligence, skill, and cultural mastery (Neel et al., 1964; Neel \& Salzano, 1967; Neel, 1980). Neel, however, did not explain the causal connections between these traits, achieving prestige and leadership roles, and obtaining wives.

Collective actions theories could potentially explain the deference of some men to other men, and the influence of some men over group decisions. Garfield et al. (2019) supported two key elements of collective action theories in a diverse cross-cultural sample - leaders increase group performance and receive payoffs but they did not strongly support one mechanism, sanctioning free-riders, although it might be that leaders instead, e.g., solve coordination problems (Van Vugt \& Kurzban, 2007). These theories do not explain why the counsel of prestigious men would often be sought. Collective action theories also do not explain why women would prefer to mate with leaders of collective actions, or those who provide other services to the group. It is possible that a wife is the "tax" or "price" charged by leaders for their services (e.g., Tiger \& Fox, 1971). As Price \& Van Vugt (2014) emphasize, however, the leader's services are a public good. If the payment for this public good is a wife, then that women is subsidizing this public good for the rest of the group, and a propensity for such purely altruistic subsidies would be selected against, contrary to evidence that women find prestigious men to be sexually attractive (Schmitt, 2014).

Male leaders' mating success could be explained by several knock-on benefits of prestige and leadership (Wiessner, 2002), such as leaders' improved ability to provision mates (Barkow, 1989), their higher genetic quality (Arden et al., 2009; Miller, 2000b), or the increased benefits they receive from group members in times of need (Gurven et al., 2000a; Sugiyama, 2004). Intelligence in mates is valued by both sexes, however, especially in long-term mates (e.g., Jonason et al., 2019, and references therein).

Another deficiency of most of these models is that they fail to consider market effects (Hooper et al., 2010 , is one exception). If every group requires only one leader or one mentor, and if every adult in the group could provide the leadership or mentorship service, then because the demand is low and the supply is high, the "price" that followers would pay for leader services might be low. The benefits to chiefs-for-a-day who coordinate work-groups (Macfarlan et al., 2012) appear to be on the low end, for instance, probably because most adults could fill this role.

The unusually high value that members of both sexes place on social and sexual relationships with knowledgeable and skilled individuals, and the disproportionate influence that knowledgeable men, but not knowledgeable women, often have over group decisions despite the lack of evidence for sex differences in intelligence, are not easily explained by any evolutionary theory of prestige or leadership.

\section{The computational services model of prestige and leadership}

Nervous systems are widely conceptualized as information processing systems - computers. These evolved to encode variable features of the environment (including features of the organism itself) as inputs to algorithms that then generate organism responses which, on average, increased the fitness of the organism (or its close genetic relatives). The brain, in short, provides a number of useful, and often essential, computational functions for the organism. Our central theses are that language enabled humans to also provide many of these computational functions to others, that these services were often extremely valuable, especially jointly improving outcomes across multiple individuals, and that there was strong selection on both sexes to provide these services, which contributed to encephalization in Homo.

Our model draws on an analogy with cloud computing. A long-standing problem in computer science is that computational resources are expensive to build and maintain, yet utilization of these resources is often 
inefficient. The computational resources must be sufficient to solve the most complex problems envisioned for the system, but because such computations will only be performed intermittently, computational resources will often be underutilized. The invention of computer network technologies enabled much more efficient use of computational resources such as mainframes, supercomputers, large computer clusters, and data centers.

The ubiquity of the internet has given rise to cloud computing: many companies offer computational services over the internet that allow customers to upload data, such as images, video, audio, or text, which the company subjects to complex computational processing to, e.g., identify faces and objects, transcribe, create a search index, or search large databases. The company then returns the output, e.g., an identity, transcription, or search result, and receives a small fee. Because a large number of companies are utilizing a large number of such computational services, standardized communication protocols have been developed. Advantages of cloud computing over traditional software distribution include that the company can continually improve its software while generating a constant revenue stream, whereas customers, instead of paying a large licensing fee up front, only pay a small fee for each use. Moreover, computational service providers often developed the software and hardware for internal use as part of their main business, but then opened up the service to others to help pay for the high costs of building and maintaining the computational infrastructure (Mell \& Grance, 2011; Armbrust et al., 2010).

We propose that because the human brain is especially expensive to build and maintain, there was a major selection pressure in the human lineage to enable individuals to maximize the fitness benefits realized from their computational capacity. In some circumstance, they would gain more fitness benefits by providing computational services to others than they would utilizing that capacity solely for individual decision-making. The communication protocol between providers and consumers was co-evolving language, although service providers also obtained substantial useful information about consumers via observations of behavior. Cultural transmission, at least when it involves teaching, is one type of computational service. So is gossiping, analyzing social situations, dispensing medical advice, and providing counsel (e.g., Hess \& Hagen, 2006, 2017). Decision-making, as we will discuss in detail, is a particularly valuable computational service. "Payments" for computational services could take a number of different forms, such as kin investment, reciprocal altruism, pseudo-reciprocity, or mutualisms. These payments subsidized the high energetic costs of building and maintaining a large brain whose computational resources might often have been underutilized. Individuals who regularly provide especially valuable computational services gain prestige.

Leadership, though, raises theoretical challenges. In sexually reproducing species, all individuals potentially have conflicts of interest. Delegating decisions to a leader puts followers at risk of exploitation. Neel (1980) admitted that he was not entirely clear how a headman achieved his position, but saw it as a gradual progression to responsibility "for the 'well-being' of 'his' village" (p. 286; scare quotes in the original). The ethnographic record of small-scale societies makes clear that prestige-style leaders are generally prosocial and achieve influence only to the extent that others permit. Nevertheless, such leaders are often highly respected and even revered, which we and many others take as evidence that they are providing valuable services to their group. We therefore operationalize a prestige-style leader as an individual that regularly makes decisions that improve outcomes for most members of his or her group, a computational service that we term joint utility improvement (JUI). We will argue that this particular service was so valuable, yet so computationally demanding, that it might help explain encephalization in Homo. We elaborate on each of these points next.

\subsection{An example computational service in non-human animals}

Predator alarm calls are a paradigmatic example of a computational service in non-human animals. Detecting a predator is an extremely computationally demanding task, involving rapid processing of high bandwidth visual, auditory and olfactory data channels. Looming visual stimuli triggers defensive behaviors across vertebrate species, including humans. Processing of such visual cues begins in the retina: retinal-ganglion cells selectively respond to approaching stimuli, activating multiple downstream targets in the amygdala and elsewhere, which then drive defensive behaviors. Auditory cues of an approaching or looming threat activate neurons in the auditory cortex, which then activates neurons in the amygdala, ultimitely triggering defensive behaviors. Meat digestion generates volatile molecules that serve as long-range cues of a predator, and predator urinary proteins act as short-range cues. In rodents, these molecules are detected by neurons in the olfactory system, which in turn activates neurons in the amygdala. Integration of multisensory cues 


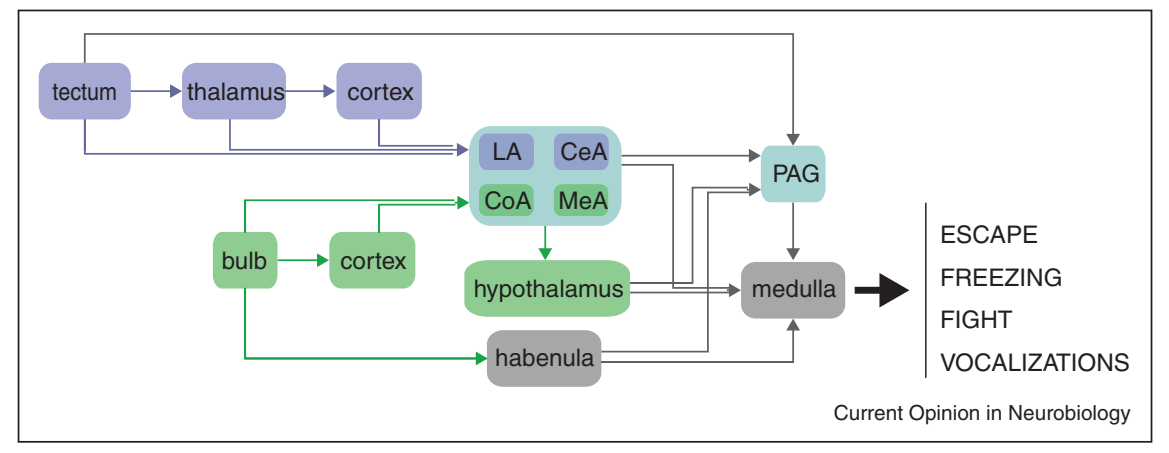

Figure 4: Generic circuit for detection of all predator cues in vertebrates. Regions in purple have been implicated in defensive behaviours triggered by visual cues, auditory or both. Green regions have been implicated in defensive responses triggered by chemical cues. Cyan regions correspond to regions that we propose to be good candidates for multisensory integration. Regions in grey are mostly implicated in gating different defensive responses. This conserved circuit reveals an interesting feature, which is that for all sensory modalities there are multiple processing stations and these can be short cut by more direct projections to the output regions. Figure and caption from Pereira \& Moita (2016). LA: lateral amygdala; CeA: central amygdala; CoA: cortical amygdala; MeA: medial amygdala; PAG: periaqueductal gray.

might occur in the amygdala and/or at the motor output level, such as in the periaqueductal gray. See Figure 4. For review, see Pereira \& Moita (2016).

The predator detection capability can be offered as a service to others. If an individual detects a predator she often gives an alarm call to warn other members of her group, perhaps saving one of their lives (e.g., Seyfarth et al., 1980; Price et al., 2015).

\subsection{The computational challenges of utility optimization}

Strategies map information states to actions (e.g., Hammerstein et al., 2006). Thus far, we have conceptualized computational services as the provisioning of useful information to others, thereby updating their information state. The recipient then performs the computations to map that information state to an action, i.e., makes a decision. Later we will argue that in humans, individuals can also provide decision-making services to others. First, though, we explain how making a decision can be extraordinarily computationally complex.

In decision theory, the individual agent has a set of decision options; a function that, for each decision, either specifies the outcome, or the probability distribution over the set of outcomes; a utility function that specifies the utility of each outcome; and an ability to determine the decision(s) with the highest utility, or, in the case of probabilistic outcomes, the highest expected utility. In some evolutionary models, utility is biological fitness, and strategies are optimized by natural selection. In other evolutionary models, computational machinery evolves under natural selection to make decisions that maximize a proxy of fitness, such as the rate of energy intake. Although we suspect that humans and other animals often utilize strategies that were optimized by natural selection, here we focus on evolved decision-making machinery that (approximately) maximizes a fitness proxy (or something similar).

Optimizing a fitness proxy can be surprisingly computationally complex. We illustrate this complexity with the famous traveling salesman problem (TSP), in which a salesman is given a list of cities and the distances between each pair of cities, and wants to determine the shortest path that visits each city and returns to the origin city. To find the shortest path with a brute-force search, the salesman would have to compute the length of each possible path through all cities. There are $n$ possible cities to visit first, $n-1$ possible cities to visit second, $n-2$ possible cities to visit third, and so forth. The number of possible paths is therefore $n$ !, the factorial of the number of cities. Hence, to discover the shortest path through only 10 cities using a brute-force search, the salesman would have to calculate the lengths of $10 !=3,628,800$ paths. For only 20 cities, she would have to calculate the lengths of $20 ! \sim 10^{18}$ paths, which is computationally intractable. 
The computational 'tractability' or 'intractability' of a problem, which is the subject of computational complexity theory, is conceptualized as the quantity of computational resources required to solve the problem. Computational resources include time (i.e., the number of computational steps), and space (i.e., the amount of memory). Researchers generally consider how quickly resource use increases as the size of the problem increases, which they denote using big $\mathcal{O}$ notation. The least complex problems require the same quantity of resources regardless of the size of the input. Determining if a binary number is even or odd, for example, takes the same amount of time regardless of the size of the binary number (just check if the last digit is 0 or 1). Problems that take constant time are denoted $\mathcal{O}(1)$. Computing the length of a single path in the TSP is somewhat more complex because the time required increases linearly with the number of cities (i.e., one must sum $n$ distances for $n$ cities), denoted $\mathcal{O}(n)$. The time to solve yet more complex problems, such as multiplying two n-digit numbers, increases quadratically with the number of digits, $\mathcal{O}\left(n^{2}\right)$, which is an example of a class of problems whose complexity increases as a polynomial function of size, $\mathcal{O}\left(n^{c}\right)$, for some constant $c$. Computer scientists generally consider a problem to be computationally tractable if the time required to solve it grows no faster than a polynomial of the size of the problem, denoting this class of problems as $\mathbf{P}$ (for polynomial time). If the time or other resource required to solve a problem grows faster than any polynomial of input size, e.g., if it grows exponentially with size, $\mathcal{O}\left(c^{n}\right)$ for some constant $c>1$, than it is considered computationally intractable. The brute force solution to the TSP grows as the factorial of the number of cities, $\mathcal{O}(n !)$, which is faster than any polynomial, and is therefore computationally intractable. It is unknown, however, if there is a more efficient algorithm that can solve the TSP in polynomial time. For an overview of computational complexity theory, see Dean (2016).

The TSP has obvious applications to optimal foraging theory, and indeed there is a sizable literature investigating if and how humans and other animals optimize total travel distance among a set of spatial locations (for a review of primate spatial foraging decisions, see Trapanese et al., 2018). There is also a growing literature that analogizes cognitive searches to searches in physical space (Todd et al., 2012; Hills et al., 2015).

When applied to group movements, the TSP helps illustrate an important distinction between the challenges posed by computational complexity vs. those posed by various types of social dilemmas. Imagine a group of 10 individuals who must decide on movement from current location A to locations B and C. All are indifferent regarding which location to visit first, but all prefer to remain together as a group. Without a leader, some individuals might travel to $\mathrm{B}$ and others to $\mathrm{C}$, resulting in low payoffs for all. By following a leader, however, e.g., the first individual to move, all individuals remain together, resulting in high payoffs for all. A leader is therefore an elegant solution to this computationally simple coordination problem.

A leader might also help solve coordination problems involving conflicts of interest. For example, assume that five of the individuals prefer to first move to $\mathrm{B}$ and then move to $\mathrm{C}$, whereas the other five prefer to first move to $\mathrm{C}$ and then move to $\mathrm{B}$, but again, all prefer to remain together as a group. In this case, a leader would travel to his preferred location first, and he and those who share his preferences would benefit most, but the other five would still receive an intermediate payoff by staying with the rest of the group, rather than receiving the lowest payoff by splitting off. Van Vugt \& Kurzban (2007) and other collective action theorists have proposed that leadership evolved to help solve such coordination problems and other social dilemmas. Notice, though, that the computational complexity of the problems is low.

In contrast, imagine a situation where a group of 10 individuals must decide the order in which to visit six locations. All prefer to minimize travel costs, and all prefer to stay together. The challenge here is identifying the shortest path among the $6 !=720$ possible paths. An individual who could rapidly and accurately identify the shortest path would be providing a very valuable computational service to her fellow group members because once the 'best' path is found, all group members have an incentive to follow it. Our computational services model includes providing solutions to coordination problems and social dilemmas, as proposed by many others, but it also includes providing 'good' solutions to problems that are computationally complex.

A novel ability to provide 'good', though not necessarily 'optimal', solutions to computationally complex strategic problems has a couple of interesting properties. First, imagine that one group member proposes a candidate path, $P_{1}$, through the six locations. Although it might be very computationally challenging to identify a shorter path than this candidate, if another individual proposes a different path, $P_{2}$, it is easy to check if $P_{2}<P_{1}$. Group members can therefore easily identify who found the shortest (but not necessarily optimal) path. Problems that might take more than polynomial time to solve, but whose solutions are 'easy' to verify, that is, can be verified in polynomial time, are termed NP (Dean, 2016). Computational services 
that provide easy-to-verify solutions will be trustworthy and therefore valued.

Second, if the ability to provide superior solutions to a class of computationally complex problems is a consequence of a fortuitous genetic mutation that alters brain function, then this skill might be difficult or impossible to be socially transmitted to others. The problem solver and her offspring who inherit this ability would be uniquely valuable to others as long as solutions to these types of problems are valued.

Finally, optimizing over the combinations of options of two or more individuals can be dramatically more computationally difficult than individual optimization. Imagine a pair of individuals that prefers to stay together, for instance, but one individual wishes to visit locations $\mathrm{A}, \mathrm{B}$, and $\mathrm{C}$, whereas the second individual wishes to visit $\mathrm{D}, \mathrm{E}$, and $\mathrm{F}$. They both want to minimize travel. If there were a path among all six locations that was similar in length to the shortest path connecting A, B, and C, as well as the shortest path connecting $\mathrm{D}, \mathrm{E}$, and $\mathrm{F}$, this would enable both individuals to stay together yet still visit their desired locations without increasing their travel costs. Finding such a path, though, could be quite computationally difficult. Finding the shortest path among three locations using brute force search only requires computing six paths, but finding the shortest path among six locations requires computing 720 paths. As we will argue in more detail later, the ability to provide solutions that jointly improve payoffs for multiple individuals, JUI, is a key computational service provided by prestige-style leaders.

The TSP is an example of a large class of discrete combinatorial optimization problems, many of which also become computationally intractable very quickly (). These, of course, are a subset of all optimization problems, which often involve finding a minimum or maximum of a function over some region of a multidimensional Euclidean space, and are also computationally difficult if not intractable for problems of even moderate size. For simplicity of exposition, however, we will generally consider discrete problems here.

Herbert Simon recognized that optimal decision-making in real humans was sharply bounded by limits on information and computing capacity. He introduced the notion of bounded rationality, the idea that real decision-makers use heuristics to solve optimization and other computationally challenging problems, often drawing on the structure of information in the environment (Simon, 1955, 1972). Simon and many others have attempted to create formal frameworks of bounded rationality, but none have gained widespread acceptance. Simon himself proposed a number of possibilities, the most well-known of which is satisficing: instead of determining the option that maximizes a payoff, the agent searches options and chooses the first that meets a predetermined threshold of acceptability (Simon, 1956). Other attempts include Selten's aspiration adaptation theory (Selten, 1990, 1998), Rubenstein's Modeling Bounded Rationality (Rubinstein, 1998), Gigerenzer and colleagues' simple, or fast and frugal heuristics (Gigerenzer \& Selten, 2002; Gigerenzer \& Todd, 1999) and the related term ecological rationality (Goldstein \& Gigerenzer, 2011), and resource rational analysis (Lieder \& Griffiths, 2019). For recent reviews, see Bossaerts \& Murawski (2017), Bossaerts et al. (2019), and Lieder \& Griffiths (2019).

Our argument is based on the following assumptions:

- 'Good' decision-making faces severe computational constraints

- Natural selection could nevertheless act on the brain so as to improve decision-making

We will use exhaustive search of options, à la the TSP, to illustrate factors that increase the computational complexity of optimization, even though real human brains are probably doing something quite different. We will therefore generally put 'optimize' in quotes to underscore that real agents evolved to make 'good' choices via decision-making processes that are currently not well understood.

\subsection{Heterogeneous but highly cooperative human groups created a rich 'market' for computational services}

Many species, including about $70 \%$ of mammalian species, do not live in social groups (Wilson \& Reeder, 2005), probably because the costs of group living often outweigh the benefits (Alexander, 1974). There

has therefore been a major effort to identify the benefit(s) of group living. In some social species, these benefits do not require much, if any, cooperation. Living in a large herd might reduce individual exposure to predators, for example, without requiring complex coordination on the part of group members. Other social species, such as social carnivores, engage in complex coordinated behaviors to achieve common goals, such as hunting big game, defending kills, competing for carcasses with other carnivores (confrontational 
scavenging), and patrolling territory (Schaller \& Lowther, 1969; Willems \& van Schaik, 2017; Stiner, 2002; Manuel \& Rayne, 2003; Brantingham, 1998; Smith et al., 2012; Bickerton \& Szathmáry, 2011).

There is a growing consensus that early members of the genus Homo were routinely engaged in carnivory by at least 2 million years ago, almost certainly as social hunters and/or confrontational scavengers (Ferraro et al., 2013; Dominguez-Rodrigo \& Pickering, 2017; Willems \& van Schaik, 2017; Brantingham, 1998; Antón et al., 2014; Bickerton \& Szathmáry, 2011). This implies that the social organization of early Homo involved complex coordination and cooperation among multiple adults of both sexes, similar to other social carnivores, which could also have been critical in defense against predators on the open savanna (e.g., Willems \& van Schaik, 2017).

Modern humans exhibit a number of interrelated life history and social traits that are rare or unique among primates, and appear to be related to our unique subsistence niche based on meat and other high quality but difficult to acquire foods (e.g., Kaplan et al., 2000). These life history traits, which include slow juvenile growth, late sexual maturity, short interbirth intervals (high fertility), high adult survivorship, and a long post-reproductive life span, ensure that human hunter-gatherer groups comprise a complex mix of individuals with widely varying levels of cognitive development, knowledge, skills, and fitness interests. See Figure 5.

In all human communities, this complex mix of individuals is organized into multiple, overlapping groups constituting biological kin, affinal kin, and non-kin. Most have what Roscoe (2009) refers to as a modular or nested structure (Birdsell, 1958; Murdock, 1949; Brown, 1991; Binford, 2001; Rodseth et al., 1991). The reproductive group (i.e., nuclear family) typically comprises a pair-bonded male and female who both invest in their joint offspring. Several reproductive units are nested within a subsistence or residential group (e.g., a hunter-gatherer band) that cooperates to acquire food (e.g., social hunting) and raise children (alloparenting). Multiple subsistence groups are nested within one or more larger groups that periodically aggregate to forage, exchange information, trade material goods, exchange marriage partners, and defend territory; these groups, in turn, typically, but not always, belong to an ethnic population that speaks a common language (Kaplan et al., 2000; Hill et al., 2011; Roscoe, 2009; Rodseth et al., 1991; Binford, 2001). Hamilton et al. (2007) found that the hierarchical structure of hunter-gatherer groups has a branching ratio of about 4: individuals are organized into families of about 4 , which are organized into bands of about 4 families, which are organized into macrobands of about 4 bands, and so forth, with about 2 more levels, resulting in a regional population of around 1000 individuals. See Figure 6. Some anthropologists deny that, among hunter-gatherers, there are levels of social organization between the band and population levels, emphasizing instead wide-ranging social networks among individuals (e.g., Bird et al., 2019). Our theory, however, only requires two levels of hierarchy: nuclear families nested within bands.

Marlowe (2005) summarized the characteristics of a large number of ethnographically known foraging societies. The median size of a band is about 30. Given the high total fertility rate of 5-6, more than half the band are juveniles, who, as we will argue in the next section, generate a high demand for computational services. Males and females can both marry out, husbands and wives commonly spend time with family of each side, and visits among, and transfers to, neighboring groups are common. Hence, the typical band comprises a fluid mix of genetic kin, affines, and unrelated adults (Hill et al., 2011; Dyble et al., 2015). See Figure 5. Given the high number of juveniles, who are 'uploading' vast quantities of environmentally-specific information, and the transient nature of band composition, there are plenty of opportunities for information sharing, a simple but important form of computational service. Bands occupy a large area (a median of over $400 \mathrm{~km}^{2}$ ), long daily trips out from camps are common (Kelly, 1983), as are residential moves (a median of 5 per year), which again create opportunities for information sharing.

At the same time, as we detail below, the diversity of band composition posed enormous optimization problems involving both computational complexities and conflicts of interest that could have jeopardized the complex cooperation that humans depended on to hunt big game and complete other tasks necessary for their survival and reproduction. See Figure 6. These problems therefore represented selection pressures for the evolution of the computational abilities necessary to solve them.

Roscoe (2009) argues that although individuals have incentives to cooperate at each level of social organization, there are also conflicts of interest at each level that could prevent cooperation. To resolve these conflicts, Roscoe proposes a signaling system. Analogous to a dominance hierarchy, individuals and groups signal physical and coalitional formidability, and then establish a ranked hierarchy so as to prevent the costs of fighting. We agree with Roscoe that a dominance hierarchy would be one way to resolve conflicts 

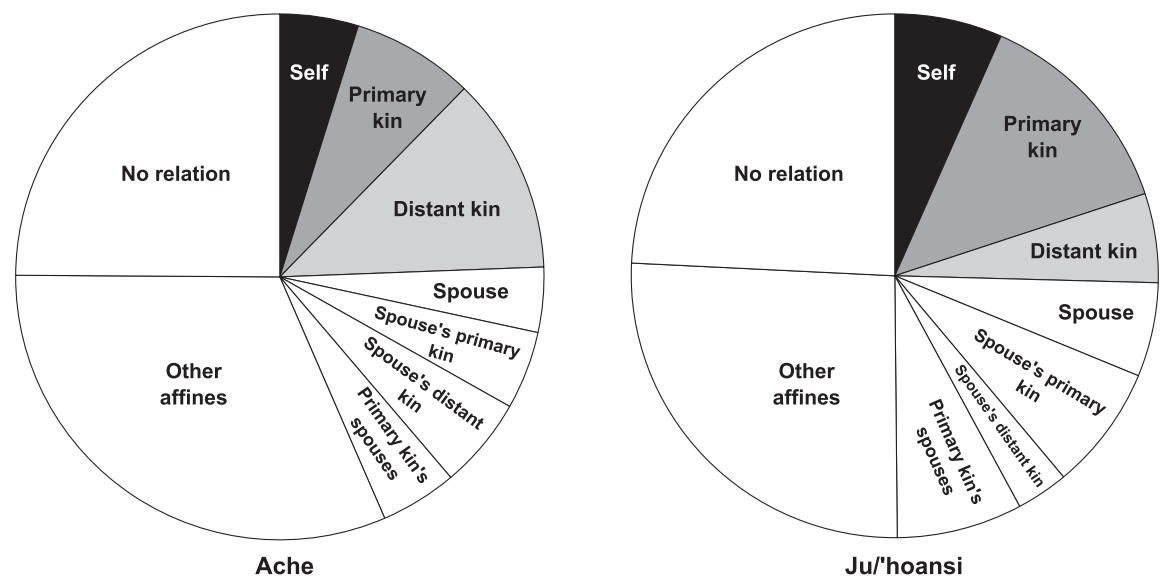

Figure 5: Mean band composition from adult ego's point of view for 58 Ache bands and $6 \mathrm{Ju} /$ 'hoansi bands (data in table S2). The category "distant affines" includes spouses of distant kin, distant kin of spouse, and affines of affines. The category "No relation" includes all adult dyads that cannot be connected in five or fewer total steps of kinship and marriage with no more than two marriage links in the chain of connection. The shaded region shows all band members genetically related to ego. Figure and caption from Hill et al. (2011).

of interest in this nested group structure. In support, Garfield et al. (2019) found that dominance-style leadership was common across cultures. But they also found that dominance-style leadership was widely disfavored and prestige-style leadership widely preferred. We therefore offer a second solution. We propose that prestige-style leadership can play a key role in maintaining cooperation at each level: reproductive groups, subsistence groups, and whatever higher-level groups that might exist that engage in collective action. Specifically, by discovering options that improve outcomes for most group members, as in our above example of finding a short path through six locations rather than only three, prestigious leaders are often able resolve conflicts at each level without imposing dominance hierarchies.

\subsection{The computational complexity of mothering}

Mothers are the archetype for our model of prestige-style leadership. We operationalized a prestige-style leader as an individual who develops a reputation for making decisions that benefit most individuals in a group. The nuclear family, or something very much like it, is a human universal (e.g., Brown, 1991). It typically comprises two unrelated parents who cooperate to raise their joint offspring, and can therefore be considered one of the fundamental units of human social organization. Mothers, we propose, were leaders of the family.

At birth, human brain size is about $400 \mathrm{ml}$, approximately one third of its average adult size of about $1300 \mathrm{ml}$ (e.g., Peterson et al., 2018). Humans consequently require substantial postnatal care. A key component of this care, we and others propose (e.g., Humphrey, 2010; Piantadosi \& Kidd, 2016), are the computational services that infants and juveniles, owing to their incomplete cognitive development, cannot yet provide for themselves. Offspring initially have limited cognitive abilities relative to adults, and little-tono environmentally-specific information. Parents and alloparents provide numerous essential computational services to these offspring, such as threat and opportunity detection, choices of food and social partners, and transmission of culturally acquired information. Mothers would be the primary providers of these services, especially for infants and young children.

Weaning time is a proxy for the helplessness of the infant (longer times indicating greater helplessness). Piantadosi \& Kidd (2016) found that, among primate genera for which data were available, there was a strong positive correlation between weaning time and an index of intelligence in adults. Thus, intelligent species have particularly helpless infants. Piantadosi \& Kidd (2016) proposed a runaway selection model for human encephalization in which big-brained infants must be born earlier so their heads are small enough 
to pass through the birth canal, which requires more intelligent mothers to successfully raise them, which selects for bigger adult brains, which selects for an even earlier birth, and so forth. In our view, however, the helplessness of infants would not increase much if they evolved to be born earlier, at least in humans compared to apes, hence the cognitive challenges of caring for them would not increase either.

We argue instead that the complexity of childcare increases dramatically when infants become toddlers, that is, when they are capable of moving independently of the mother. At this point a mother must compute her child's position and actions relative to dynamically changing threats posed by numerous features of the environment, such as bodies of water, large drops, dangerous animals, toxic plants, and social antagonists, and quickly intervene, if necessary, a computational service that is a generalized version of the predator alarm calls we discussed earlier. She must also identify social and other opportunities for her child. These computational services require accurate inferences about many aspects of the child's state, e.g., hungry, sick, scared, happy, interested, or bored, a capability often referred to as Theory of Mind (for a recent review and critique, see Schaafsma et al., 2015).

Women in natural fertility populations have relatively short interbirth intervals compared to chimpanzees, yet human children require provisioning for up to 18 years. A mother is therefore typically caring for multiple children, which multiplies the complexity of the computational challenges she must solve. Moreover, dependent offspring of widely varying ages have substantially different needs: infants must be carried and nursed; toddlers and children must be fed, supervised, and trained in numerous domains (e.g., foraging skills); and adolescents that are sexually maturing and beginning to compete for mates must be advised (Kramer, 2010). Unlike other primates, humans of both sexes also maintain lifelong relationships with offspring of both sexes (Rodseth et al., 1991). Mothers must therefore develop, maintain, and update a cognitive model of each child, supplying a constant stream of child-specific computational and other services for the rest of their lives. Recent systematic evidence from a small-scale and developing societies reveal that autonomous decision making by mothers is positively associated with better nutritional outcomes in offspring (e.g., Starkweather \& Keith, 2018; Engle, 1991). It is possible that entering the human 'niche' of short interbirth intervals combined with a long period of juvenile dependency required a substantial increase in human female cognitive capacity relative to our ape ancestors.

\subsection{Joint utility improvement}

One of the most important computational services mothers provide their juvenile offspring are the numerous decisions they make for them, such as choices of food, social partners, and learning opportunities. Because a women and her children are only related by $r=0.5$, however, their interests are not perfectly aligned (Trivers, 1974; Mock \& Parker, 1997; Royle et al., 2012). Thus, a decision that might optimize an outcome for a child might not not optimize the outcome for the mother, and vice verse. Mothers constantly face the challenge of making decisions for offspring that improve child outcomes while at the same time improving their own outcomes, an example of joint utility improvement (JUI).

Because JUI involves searching not only among one's own options, but among combinations of all those options with all the options of one or more other individuals, it is dramatically more computationally complex than optimizing an individual outcome. In typical game theory analyses of multi-agent conflicts, a small number of options are considered for a small number of players. In the one-shot Prisoner's dilemma (PD), for instance, there are two players with two options, for a total of four different possible payoffs for each player. In real life, though, there are usually many more than two options for each agent and many more than two agents. A typical hunter-gatherer mother has four children. If, in any given family situation, the five of them each has only 10 options, there are $10^{5}$ possible payoffs for each. The complexity of a brute force assessment of possible payoffs to find the optimum via cognitive processes (rather than via natural selection) is $\mathcal{O}\left(c^{n}\right)$ in the number of group members, which is computationally intractable for all but the smallest $n$.

Because exact optimization is unlikely to be computationally tractable, we emphasize improvement of utility. That is, however brains can improve outcomes for the individual, they can also improve outcomes for multiple individuals, albeit at the cost of greater computational complexity. If we are correct, JUI was a major selection pressure on female cognitive abilities.

Mothers would routinely use JUI abilities to make good decisions for their cognitively undeveloped offspring, but they could also use JUI abilities to help resolve family conflicts by enticing antagonists towards options with higher payoffs, and thus simultaneously improving their own outcomes. Consider two young 


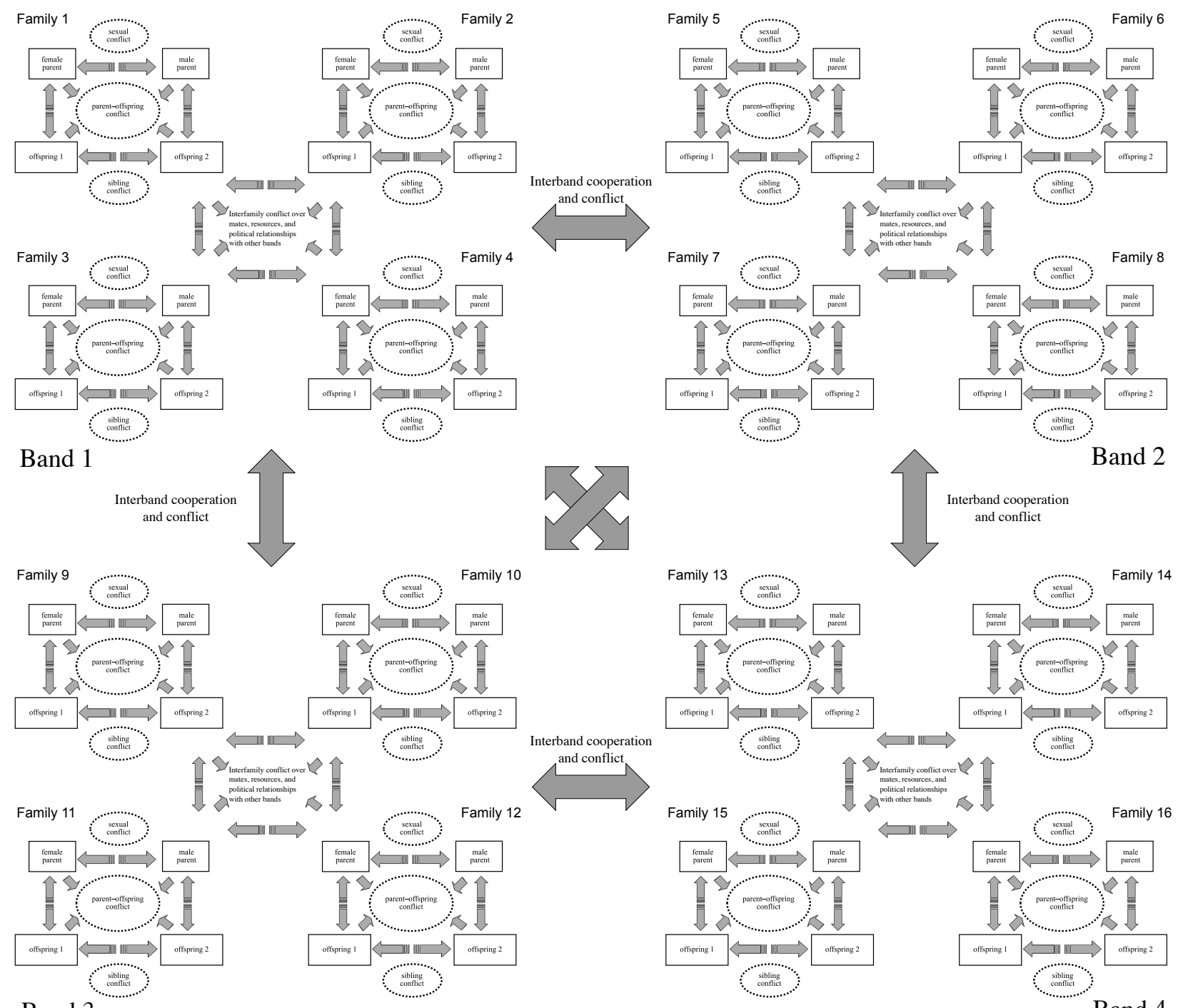

Band 3

Figure 6: Typical hunter-gatherer bands. There are intra- and inter-familial conflicts over parental investment, mates, and resources. Siblings compete over the investment from parents (sib competition). Offspring have different interests from their parent(s) over investment (parent-offspring conflict). Where both parents invest, they are in conflict over the amount each should give (sexual conflict). Members of different families cooperate within bands, but nevertheless compete over resources, and adult members compete over access to mates. Bands cooperate and compete over territory and game. Figure and caption modified from Parker et al. (2002). 
siblings who are fighting over a toy. As any parent knows, one good solution to this problem is to entice one or both children to do something else. Assume that each child has 5 options, with the utility of each option depending on what the other child does. The utility of choosing the toy, for example, if each makes that same choice, is lower than if only one does. ${ }^{2}$ To entice the children to other activities and stop the squabbling, the mother must first have developed cognitive models of each child's options and utility functions. Minimally, she must then quickly evaluate $2 \times 5^{2}=50$ possible payoffs, identifying options for each child that, if not optimal, at least improve the payoff for one child without decreasing the payoff for the other (a Pareto improvement). The mother reminds one child that her friends are playing outside. Since playing with friends has higher utility than playing with the toy, that child leaves, resolving the conflict.

Why didn't that child make that choice herself without the mother's intervention? Perhaps the mother had information the child did not. But it could also be the case that child did not have the cognitive capacity to evaluate all the options, especially if her attention was focused on the conflict with her sibling. Perhaps she only evaluated the options involving other toys, or that they could both play outside, but she didn't consider the option that she could leave while her sibling stayed inside. An important property of the mother's proposal that one child play with the toy and the other play outside is that although it might have been computationally difficult to discover, it is computationally much easier for each child to verify that their payoffs would increase since they do not need to search all combinations of options but simply compare the payoffs for this single set of options to their current payoffs.

The mother, though, must evaluate not only combinations of her children's options and corresponding payoffs, but additionally combine them with her own 5 options and payoffs $\left(3 \times 5^{3}=375\right.$ payoffs $)$, avoiding options for her children that reduce her own payoff. For example, during her cognitive search of her children's options, perhaps she discovered that she could entice them with food she was cooking. However, these children had recently eaten, whereas her other two children had not, and she wanted to save the food for them. She therefore deliberately did not offer the food option because it would have reduced her own payoff. The mother therefore used her superior cognitive abilities and access to information to provide her children a valuable computational service that increased their payoffs. At the same time, she increased her own payoff by ending the squabbling and also by diverting the children from an option - begging for food - that would have reduced her payoff.

In addition to the mother and father, human infants and young children have many other caretakers alloparents - which include their older siblings, cousins and grandparents, and their parent's siblings and friends. Alloparenting is so important to childrearing that some authors describe humans as cooperative breeders (Meehan et al., 2016; Hrdy, 2011, 2006; cf. Bogin et al., 2014). Across diverse species, cooperative breeding is associated with increased prosociality and greater capacities for social cognition and cooperation (Burkart et al., 2009). To the extent that alloparents are performing the same childcare tasks as mothers, and to the extent that alloparents benefit from their alloparenting, the alleles for JUI and other computational services will also be positively selected in alloparents. Alloparenting also creates additional computational challenges for the mother. Mothers no doubt evaluate alloparents for their skills and the degree to which their fitness interests align, and because some alloparents belong to other families, alloparenting creates ties among families in subsistence groups. Thus, alloparenting requires mothers to manage complex within- and between-family relationships (e.g., Hrdy, 2011).

Although the brain nears maturity in late adolescence, development of some brain regions continues into early adulthood (Lebel \& Beaulieu, 2011). Hence, even adolescents and young adults might benefit from their parents' mature decision making mechanisms and greater experience. In many hunter-gatherer and other traditional societies, for example, parents play key roles in their offsprings' long-term mate choices, e.g., arranged marriages (Walker et al., 2011). To illustrate: three suitors might seem to be equally good from a daughter's perspective. The mother, however, might detect a serious flaw in suitor 1 . The other two suitors are indeed equally good for the daughter, but suitor 2 would result in a low payoff for the mother he and her brother don't get along. The mother steers her daughter toward suitor 3, who indeed produces a high payoff for both daughter and mother.

In summary, based on her increasingly detailed cognitive models of the preferences and capabilities of her children, her superior knowledge, and her superior computational abilities that enable her to rapidly search a larger space of options, the mother can make decisions for her children that result in higher payoffs

\footnotetext{
${ }^{2}$ Or perhaps it is higher!
} 
than if the children made their own decisions. In addition, she manages alloparents, and can help resolve conflicts, or avoid future conflicts, within and between families by enticing antagonists towards activities or choices that increase everyone's payoff. JUI and other computational services, we propose, characterize prestige-style leaders at all levels of human social organization.

\subsubsection{Female leadership in non-human animals}

There has been an increased focus on processes of leadership and followership among nonhuman species within biology in recent years (e.g., Garland et al., 2018; Smith et al., 2018, 2016; Koykka \& Wild, 2015; Strandburg-Peshkin Ariana et al., 2018). The dominant framework of leadership behavior among nonhuman species, which focused primarily on group movements (e.g., Couzin et al., 2005; Guttal \& Couzin, 2011), has now been extended to include multiple domains of leader-follower behaviors, including in collective actions, foraging strategies, and the roles of leaders in within-group conflict resolution and inter-group conflicts (Smith et al., 2016). Ethologists have long highlighted a few model species that exhibit patterns of female leadership, distinct from other closely related species, such as spotted hyenas (Boydston et al., 2001; Henschel \& Skinner, 1991), bonobos (Furuichi, 2011; Tokuyama \& Furuichi, 2017), and killer whales (Brent et al., 2015).

Smith et al. (2018) constructed a database of mammalian species known to exhibit leadership in at least one of four domains: collective movement, foraging, and conflict management both within and between groups. Their sample included 76 non-human species across eight orders that exhibit some leadership behaviors in some contexts, where leadership was defined as cases where individuals have a disproportionate level of influence over collective behaviors in one of the aforementioned four domains. They then constructed a phylogeny of female-biased leadership, defined as cases when females exclusively lead collective behaviors or occupy leadership roles more frequently than males on average in one of the three domains (contrary to our perspective, collective movement was excluded to avoid a focus on mothers leading dependent offspring). ${ }^{3}$

Results revealed eight of the 76 mammalian species, roughly $10 \%$, demonstrated female-biased leadership, which suggests the near universal male bias in community leadership seen among diverse human societies is a pattern consistent with vast majority of diverse non-human mammalian species (Smith et al., 2018). For several species, strong female leadership occurs in the context of female-dominated societies, such as among spotted hyenas and lemurs, where an individual female is able to outrank an individual male. In these rare cases, there is a lack of, or reversal of more typical sexual dimorphism, in that females are not significantly smaller or are larger than males. Smith et al. (2018) therefore suggest smaller physical size is likely a strong constraint on the emergence of female-biased leadership among nonhuman animals. Strong female-biased leadership may also be more likely to emerge when groups of related females form strong alliances and may also be more likely to emerge among older individuals (Smith et al., 2018).

Their cross-species analysis carries implications for female leadership in human societies. Consistent with Garfield et al. (2018), Garfield et al. (2019), and our arguments here, women may be more likely to exhibit leadership roles (1) within families and more local organizations, (2) when groups of women form cooperative alliances, (3) later in life as repositories of cultural knowledge, (4) in conflict resolution, and (5) taller more physically formidable women may be more likely to exhibit strong leadership in various contexts (Smith et al., 2018).

\subsection{Prestige-style leadership beyond the family}

Given the nested structure of human societies, JUI and other computational services that are valuable within the family will also be valuable within the subsistence group and within larger regional security/ethnic groups, and the autosomal alleles for computational services, including JUI, that provided benefits to mothers would also have provided benefits to males. In humans, males invest in offspring too, although they typically

\footnotetext{
${ }^{3}$ Smith et al. (2018, pp. 4-5) explain their rationale as follows: "Here we focused on key cases for which female-biased leadership transcended into at least one additional domain beyond group travel. We adopted this approach because the role of female leaders within the domain of travel has been discussed extensively elsewhere and because we seek a more holistic view of the traits of female leaders who occupy leadership roles in domains for which male-biased leadership is most evident in humans. Briefly, female leadership during travel is most often explained by dependent young [e.g., seeking nutrition from nursing, (Fischhoff et al., 2007)] and/or less informed individuals who follow elder females [e.g., who presumably possess enhanced local knowledge (Brent et al., 2015)]."
} 
provide less direct care of infants and young children than do females. Aka fathers provide more infant care than fathers in any other known society, for example, and yet still only provide $22 \%$ of care (Hewlett, 1993). Direct paternal involvement with offspring increases as offspring mature, however.

Many of the opportunities to provide computational services within families, though, also exist beyond the family. Earlier we provided the example that finding a short path through six locations could resolve a conflict between two individuals that have different travel preferences. Both men and women can offer computational services to fellow band members, such as providing valuable information and good solutions to complex optimization problems. Most leaders beyond the family appear to be men, however, perhaps because women in their reproductive years are focused primarily on leading the family. Older women, however, often do assume supra-family leadership roles (Garfield et al., 2018).

Mobile hunter-gatherer bands typically comprise 4-6 families. There will be informational asymmetries and difficult-to-solve optimization problems involving group movements, foraging, conflicts among the adults, and political relationships with other bands. It is possible that, to reduce computational complexity, decisionmakers at supra-family levels make the simplification that families can be considered as single agents. Hence, optimizing decisions for a typical hunter-gatherer band would involve optimizing outcomes for four 'agents' rather than 16. Similarly, optimizing outcomes for four bands would again involve optimizing outcomes for four 'agents' rather than 16 families or 64 individuals. Alliances and conflicts among bands, especially lethal conflict (warfare), might have posed especially strong selection pressures on decision-making abilities because individuals at the high end of the JUI spectrum are competing with their counterparts in other groups (Flinn et al., 2005; Alexander, 1990; Gavrilets \& Fortunato, 2014; Choi \& Bowles, 2007; Bowles, 2009; Chagnon, 1988). See Figure 6.

Garfield et al. (2019) found that leaders were described as intelligent and improved group performance and payoffs in about 70-75\% of cultures, and as having expertise, providing counsel, and being respected in about $85-90 \%$ of cultures. Discovering options that simultaneously increased the payoffs of most group members was computationally demanding, potentially involving a search over combinations of all options of all individuals. We propose that a defining characteristic of prestige-style leaders is that they used their greater knowledge, nuanced cognitive models of group members, and computational abilities to discover such options.

\subsection{Profiting from computational services}

Computational services can be extraordinarily valuable. Some, like predator alarm calls, can be lifesaving. Social animals often give alarm calls when other group members are close kin (e.g., Sherman, 1977; there can also be individual benefits to vocalizations, e.g., Sherman, 1985). Hence, the "payment" for giving the alarm call is increased inclusive fitness. Many of the computational services mothers provide their offspring would be 'paid for' via inclusive fitness benefits. Reciprocal altruism is another mechanism for receiving payments for computational services (Price \& Van Vugt, 2014), although to avoid free-riding, services could probably not be public goods.

Connor (1986) introduced a cooperative strategy, termed pseudo-reciprocity, that is much more likely than reciprocal altruism to evolve in wide range of species. In pseudo-reciprocity, one agent provides a benefit to another agent purely as a byproduct of its own self-interested actions; the recipient of this benefit therefore invests in the provider so as to maintain or increase its access to the benefit. A tree grows branches and leaves to feed itself, for example, but this incidentally provides shade to a homeowner. The homeowner values shade, so she regularly waters the tree at a cost to herself that is less than the benefit of shade. There is no incentive for the tree to 'cheat', and therefore no need for the homeowner to evolve cheater detection mechanisms. Nor is there any need for repeated interactions or the evolution of mechanisms for individual recognition (see also Leimar \& Connor, 2003; Leimar \& Hammerstein, 2010; Roberts, 2005; Connor, 1995; Queller, 2011). Queller (2011) refers to this and other cooperative relationships among non-kin as kith selection. See Figure 7.

Tooby \& Cosmides (1996) and Henrich \& Gil-White (2001) apply pseudo-reciprocity to friendship and prestige in humans (see also Sugiyama, 2004; Gurven et al., 2000b). Tooby \& Cosmides (1996), for example, write:

Behaviours that are not undertaken as intentional acts of altruism often have side-effects that are beneficial to others - what economists call positive externalities. Some potential associates 


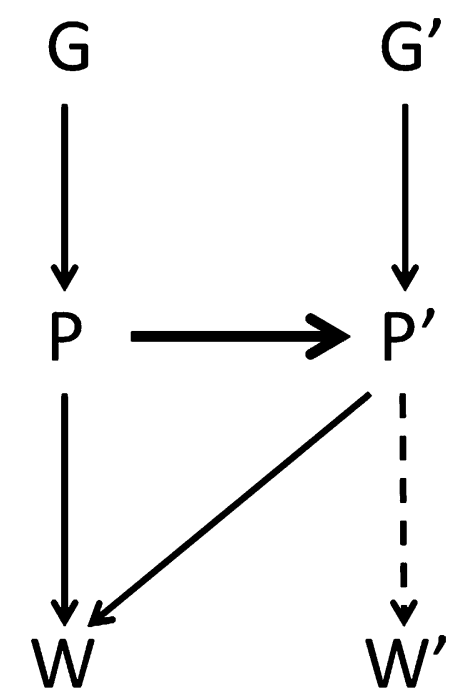

Figure 7: Kith selection. An actor's phenotype $P$ can influence $P^{\prime}$, its partners phenotype (often for a different trait), by manipulation, partner choice, and partner fidelity feedback (heavy arrow). These components create an association between phenotypes $P$ and $P^{\prime}$ and therefore, also between $P^{\prime}$ and $G$. Figure and caption from Queller (2011). [ $G$ and $G^{\prime}$ are the genotypes of the actor and its partner, respectively; $W$ and $W^{\prime}$ are the respective fitnesses.]

exude more positive externalities than others. For a knowledge-generating and knowledge intensive species such as ours, such situations abound. Someone who is a better wayfinder, game locator, tool-maker, or who speaks neighbouring dialects is a better associate, independent of the intentional altruistic acts she might direct toward you. Similarly, there are an entire array of joint returns that come about through co-ordinated action, such as group hunting or joint problem-solving. Individuals may vary in their value as friends and associates because they contribute to the general success, or because their attributes mesh especially well with yours or with other members of your cooperative unit.

Henrich \& Gil-White (2001), as we discussed earlier, similarly argue that the phenomenon of prestige is precisely the deference and other forms of investment of some individuals provided to other individuals from who they can obtain particularly valuable knowledge and skills. Pseudo-reciprocity is a plausible mechanism for profiting from computational and other services. Individuals will defer to, and otherwise invest in, those who provide them valuable but private computational and other services. In social groups that depend on close-knit cooperation to survive and reproduce, JUI would be particularly valuable because it could help prevent costly conflicts and group fissioning.

In addition to receiving investment from those who value their services, computational service providers can also invest in consumers to influence them in ways that benefit the providers. As in our examples of squabbling children and providing mating advice, a service provider might discover an option for an advisee that increases payoffs for both. A well thought out plan for a cooperative venture might inspire multiple individuals to participate, to the benefit of all. The incentive for the leader to make decisions in the interests of a follower, then, is that, given multiple outcomes with approximately equal payoffs for the follower but unequal payoffs for the leader, the leader would be able to influence the follower to choose the option with the higher payoff for the leader.

An agent that surrenders its decision-making to another agent, though, puts itself at risk of exploitation. There is substantial evidence from social psychology that followers are particularly sensitive to equitable outcomes when relinquishing decision-making authority to other agents or institutions (Bøggild \& Petersen, 2016, and sources cited within). As followers experience the outcomes of successive decisions by a leader and the associated costs and benefits, they are likely to continually and facultatively adjust their level of support for the leader. The concept of a welfare tradeoff ratio suggests followers will withdrawal support from a 
leader with a disproportionate level of influence when the costs and risk of exploitation outweigh individual benefits (Petersen et al., 2010; Tooby et al., 1996).

Followers had at least two safeguards against such exploitation. First, JUI problems might be in NP. That is, although solutions are computationally difficult to discover, it might be relatively computationally simple for a follower to verify that a solution indeed increases his own payoff. Second, if a leader's decision did not result in favorable outcome for the follower, i.e. the welfare tradeoff ratio became unfavorable, the follower could abandon the leader, and the leader would lose the opportunity to influence that follower's future decisions in ways that benefit the leader.

\section{Computational services, sexual selection, and biological markets}

Sexual selection theories of prestige and leadership (e.g., Tiger \& Fox, 1971; Barkow, 1980; Neel \& Salzano, 1967; Neel, 1980) rest on the clear evidence of biased reproduction of male leaders and other prestigious men. Other than Barkow (1980), though, most of the evolutionary theories of prestige and leadership do not specify what these benefits are. Given that for every man with two wives another man has none, it must also be the case that other men also place a high value on the benefits provided by prestigious men.

It's not clear, however, that the putative benefits delivered by leaders and prestigious men are particularly valuable. The Henrich \& Gil-White (2001) cultural transmission theory, for example, ignores the mating success of prestigious men. Moreover, although some socially transmitted skills like hunting might merit deference to particularly skilled individuals, many other important examples of cultural transmission do not seem to merit such deference. Language is one of the most valuable socially learned skills, yet it can be acquired from almost any member of the group, including children. Identifying edible plants is another critical socially learned skill that also can be learned from most members of the group. Furthermore, it is implausible that women are choosing prestigious men as mates so that they can better learn from them (nor do Henrich and colleagues make any such claim).

The value of leaders of collective actions also might not be particularly high. Leaders of work groups (chiefs-for-a-day), for example, do not seem to have much prestige (Garfield et al., 2019), probably because only one leader is needed and most adults could fill this role. The service-for-prestige model (Price et al., 2014; Price \& Van Vugt, 2014) is similarly vague about the value of the services to members of the same and opposite sex.

We take the reproductive success of leaders and other prestigious men as evidence that they provided substantial benefits to mates that were not available to the same degree from other men. Intelligence is a key trait of prestige-style leaders across cultures (Garfield et al., 2019). Intelligence is also a highly ranked mate preference for both sexes (Prokosch et al., 2009; Lee et al., 2014; Jonason et al., 2019; although among the Hadza, a hunter-gather society, women preferred intelligence in a mate more than men; Marlowe, 2004). These facts support Neel's theory of leadership. But what, exactly, were the benefits of intelligence and/or leadership status, to mates?

The JUI hypothesis explains why women would prefer mates who are good JUI decision-makers, above and beyond their ability to provide more resources (Barkow, 1980) or good genes (Miller, 2000a). Long term mateships are not so different from any other cooperative group that benefits from leadership abilities: they involve cooperation by two unrelated individuals to raise their joint offspring over a period of perhaps 20 years or more, but who also have numerous potential conflicts over, e.g., investment in offspring from previous mateships, investment in the genetic kin of one mate vs. kin of the other mate, and other mating opportunities. Divorce rates, not surprisingly, are high among both small-scale and large scale societies (Hewlett, 1991; Blurton Jones et al., 2000). Women who marry men that excel at good decision-making in general, and finding JUI solutions in particular, will avoid costly conflicts and otherwise enjoy better outcomes, and thus have higher fitness than other women. By the same token, men who marry women that are superior decision-makers will have wives that make good decisions for their offspring, avoid family conflicts and otherwise achieve better outcomes, and will thus have higher fitness than other men.

JUI abilities would depend on many genes, some of which would suffer deleterious mutations and, much more rarely, beneficial mutations. The development of these abilities would also probably be sensitive to nutrition and other environmental conditions. JUI abilities, like many complex traits, would therefore vary significantly among adults, and individuals with high JUI abilities would be in short supply. Since most 
reproductive-age adults seek long-term mates, there would be high demand. Services at this level also do not face a public goods problem. Hence, the JUI hypothesis predicts sexual selection for JUI adaptations in both men and women. Because polygyny would involve optimizing outcomes for the husband and, e.g., two wives, success in such relationships might require one or more participants with exceptional abilities at JUI. Finally, and perhaps most importantly, because group decisions at higher levels of social organization would more reliably align with the interests of group leaders and their families than with those of other group members, marrying a leader would minimize costly conflicts between group decisions and one's own fitness interests.

Mating markets are just one example of the more general phenomenon of biological markets, in which partner choice and trade are important selective forces (Noë \& Hammerstein, 1995; Hammerstein \& Noë, 2016). Our computational services model incorporates key insights of biological market theory (BMT), including:

- competition among biological traders can reduce the 'price' of a commodity (the price need not be the same for all individuals) and it can lead to specialization.

- supply and demand can influence the net benefits from a trade.

- traits can evolve solely because of market selection induced by partner choice in trade-like situations (an analogy with sexual selection).

- the market can limit the scope for false advertising.

- trading partners can be controlled by the threat of partner switching.

Demand for JUI services relative to supply might or might not be high at the level of the subsistence group. On the one hand, the group only needs one leader in any particular context. Although JUI abilities would vary, all adults would possess the psychological adaptations for JUI, so supply would be relatively high relative to demand. On the other hand, prestige-style leaders have to be able to find JUI solutions that are not easily discovered by other group members. That is, as envisioned by Neel, prestige-style leaders would be at the upper end of a distribution of JUI abilities, and so supply might be limited. Given that such services are public goods (Price \& Van Vugt, 2014), it could be that group members nevertheless do not pay leaders in small-scale societies for these services. Instead, leaders might profit by aligning group decisions with their own fitness interests (cf. Gavrilets, 2015). A follower might show deference to a leader, however, in part, for the private benefit that the leader would adequately weight the follower's interest when seeking group-beneficial solutions, as it would probably be rare that decisions perfectly optimize outcomes for all group members, yet switching groups is costly.

\subsection{Personalized computational services}

Some computational services, such as predator warnings, would be useful to everyone. To maximize their value, many computational services would need to be tailored to the consumer, however, which means that providers must develop and maintain a cognitive model of each consumer. Some services might be most useful to individuals of particular ages or sexes. Teaching identification of edible plants would be most useful to young children, for example, whereas teaching hunting skills would be most useful to older children and young adults. Yet other services would be most useful to individuals in particular states, such as ill, angry, sad, hungry, and so forth. Humans have a rich array of universal facial expressions and other signaling mechanisms to convey exactly this information to social partners (e.g., Ekman, 2016).

\section{Cognitive architecture and encephalization}

Given that there is a universal human nature, it is possible that, to deliver personalized computational services and predict behavior, humans have evolved a generic cognitive model of social partners whose parameters could be set for each partner using readily available information, such as cues of age, sex, and state. Beyond this, there would be parameters that are specific to individuals, such as their social 
relationships, capabilities, preferences, physical locations, and so on. Moreover, this model could also be used to optimize decisions for oneself. In other words, one possible architecture for providing computational services is that the cognitive mechanisms that an individual uses to process information for herself could be activated using parameters for someone else, thus generating outputs that are useful to them. Such capabilities are commonly referred to as theory of mind (ToM) and perspective-taking (Premack \& Woodruff, 1978; Schaafsma et al., 2015; Martin \& Santos, 2016; Underwood \& Moore, 1982; Lamm et al., 2007, and references therein).

ToM-like abilities undoubtedly require additional neural circuitry, especially memory to store parameters for all the individuals in one's social network. Facial recognition alone might require a marked increase in memory circuits, as human hunter-gatherers typically interact with more than three hundred same-sex adults during their lifetimes, and about a thousand individuals when opposite-sex adults and children are included (Hill et al., 2014; Wiessner, 2014). Cultural transmission, a key computational service, also required an increase in brain size to provide more memory to store culturally transmitted knowledge (Muthukrishna et al., 2018). As a long-lived, wide-ranging species, humans would also have benefited from greater memory capacity to individually learn from their substantial exposure to temporal and spatial variation, and to use this knowledge to improve individual decision-making and to provide valuable computational services. Increased memory resources would have contributed to encephalization.

Neel's original insight, though, was that sexual selection for leadership qualities might help explain encephalization in Homo (Neel \& Salzano, 1967; Neel, 1980). The JUI hypothesis might help explain the evolution of a qualitative increase in computational abilities in Homo. In standard decision theory, the agent finds her optimum choice among all possible combinations of choices of all agents, full stop. This ideal is computationally intractable. Real agents probably use a number of heuristics and other techniques to sharply limit the search space, as reviewed in section 6.2. If a JUI-agent uses her computational machinery with parameter values of another agent to discover a good option for them, she will often find a different option than the one that is best for her. The JUI-agent will then have to compare her best option with this alternative. In the likely case that there is a conflict of interest, the JUI-agent will have to engage in a search of combinations of options, seeking one that is attractive to both agents, which, as we have seen, can dramatically increase computational complexity.

JUI solutions would only be useful, though, if they could be discovered in a reasonable amount of time. One strategy to reduce computation time is to divide the problem into several smaller sub-problems that can each be solved by multiple processing units operating simultaneously, i.e., parallel computing. Using 10 processors to find the shortest path through 10 cities, for example, each searching all paths that start with one of the 10 cities, would reduce search time by a factor of 10. Many computational neuroscientists believe the brain comprises multiple processors operating in parallel (e.g., Alexander \& Crutcher, 1990; Nelson \& Bower, 1990; Zeki, 2015). Selection for increased parallel processing could have contributed to encephalization by increasing the number of neural circuits in the brain, which might explain the connection between increased social complexity and increased brain size (Barton \& Dunbar, 1997).

\section{Theoretical challenges and caveats}

The notion of 'computational service' has fuzzy boundaries because most services that one person provides another rely, at least to some extent, on the brain. We therefore restrict this notion to services that accrue most of their value from the information possessed, and computations performed, by the service provider, and that are typically provisioned via signaling of some sort, usually language, but also other types of vocalizations and gestures, and whose effect is to improve the decision-making of the recipient. We relax the language requirement for services provided to children who have not yet acquired language. Grabbing a toddler before he stumbles into the fire, for instance, or puts a poisonous berry in his mouth, would be categorized as computational services because their value comes primarily from information and computations possessed and performed by the caretaker, and their effect is to alter the toddler's decisions, to his benefit. Sharing foraged foods, however, would not be so classified because although obtaining wild foods requires considerable knowledge and computations, the value of the service is primarily the calories in the food, and not improved decision-making by the recipient. We agree with Tiger \& Fox (1997), Barkow (1980), Price \& Van Vugt (2014), and many others, that non-computational services, like hunting and sharing food, 
can increase prestige. We do not offer the computational services model as a comprehensive explanation of prestige, however, but only as an explanation for the role of knowledge and intelligence in prestige.

The connection between computational services and language raises a chicken-or-egg problem. Was the selection pressure to provide computational services the impetus for the evolution of language? Or did language evolve first, and the ability to provide computational services follow? The literature on language evolution is voluminous, but roughly falls into two camps: language as an instrument of communication or language as an instrument of thought (e.g., Bolhuis et al., 2014; Corballis, 2017; Everaert et al., 2017; Pinker \& Jackendoff, 2005); some, like Carruthers (2002), strike a middle ground. The computational services model is obviously in the communication camp. We do not (yet) offer the computational services model as a general explanation for the evolution of language. We do think, though, that improving the delivery of computational services might have been one selection pressure on language.

The JUI concept also raises several issues. Virtually all game theory models assume that agents aim to optimize their own outcomes given the choices made by other agents. JUI, however, proposes that agents sometimes seek options that optimize outcomes for multiple agents. It is therefore important to clarify what JUI is not. It is not a general solution concept like a Nash equilibrium or an evolutionary stable strategy (ESS), nor is it a group-beneficial strategy. Instead, it is an individually beneficial strategy that is employed by agent $\mathrm{A}$ to entice agent $\mathrm{B}$ to act in agent A's interest. It would typically be used when agent A has superior information and/or computationally abilities relative to agent B. Informational asymmetries would routinely exist among parents and their offspring, among older vs. younger generations, and among adults. Computational asymmetries, on the other hand, would exist between all adults and cognitively immature children, but would only exist between some adults.

JUI also requires that there are, in fact, options for agent B, unknown to B itself, that would increase payoffs for both agents, and are discoverable by A. This is a strong requirement that would commonly be met with parents and their young offspring, but might be much more difficult to meet among adults, especially if agent $\mathrm{B}$ has a high-payoff alternative and/or is likely to discover the option itself. Hence, agent A must balance the computational resources (time, memory, opportunity costs) required to search for a JUI solution against the likelihood of finding one and the costs to her if she doesn't. Agent A might also be competing against agents $\mathrm{C}, \mathrm{D}$, and $\mathrm{E}$ to provide a solution to agent $\mathrm{B}$. In the event that JUI solution is not to be found, and conflict ensues, other strategies would then be used to manage it, such as aggression or switching groups.

Group living is obligatory for humans, however, and aggression and switching groups can be costly. For the nuclear family group, switching costs are particularly high. For a given offspring there are only two adults who are highly genetically related to it and could therefore realize substantial fitness benefits by cooperating to raise it: the two biological parents. ${ }^{4}$ Offspring can't switch biological parents, nor can parents switch offspring without paying the cost of pregnancy. If both parents are alive, the benefits of finding JUI solutions to enable cooperative childrearing would be substantial.

The JUI hypothesis also faces the chicken-or-egg problem faced by most social intelligence theories: did complex societies select for larger brains? Or did a larger brain, which evolved for some other reason, enable the formation of complex societies? Social intelligence theorists favor the former, obviously, but we remain agnostic. Early Homo was an exceptional primate in many regards: it was large-bodied and bipedal, made sophisticated stone tools, incorporated a substantial amount of vertebrate meat in its diet, and spread throughout much of the Old World. Its modern descendents in natural fertility populations have short interbirth intervals, high fertility, and rely heavily on alloparents. Many theories of encephalization that focus on one or more of these traits remain in play. At a minimum, our computational services model provides a mechanism for a language-using species to make more efficient use of a large, energetically expensive brain. We agree with Neel, though, that sexual selection of intelligent leaders is a plausible factor in human encephalization.

\section{Concluding remarks}

The author of The Prince, a work that often prescribed rule by fear and force, dedicated his book to his prince with the following words:

\footnotetext{
${ }^{4}$ In some cases, a much older sibling could help raise an infant.
} 
Those who strive to obtain the good graces of a prince are accustomed to come before him with such things as they hold most precious, or in which they see him take most delight; whence one often sees horses, arms, cloth of gold, precious stones, and similar ornaments presented to princes, worthy of their greatness.

Desiring therefore to present myself to your Magnificence with some testimony of my devotion towards you, I have not found among my possessions anything which I hold more dear than, or value so much as, the knowledge of the actions of great men, acquired by long experience in contemporary affairs, and a continual study of antiquity; which, having reflected upon it with great and prolonged diligence, I now send, digested into a little volume, to your Magnificence.

Machiavelli, whose utmost concern was the survival of the state, thus presented himself as a wise consigliere, perhaps even as a prestige-style leader of princes. His counsel is clear: This, your Magnificence, is what you must do to preserve the Florentine Republic. Machiavelli's prestige has endured across the centuries. He was also notorious for his numerous extramarital affairs. He thus embodied the two cornerstones of Neel's theory - leadership based on superior knowledge and intelligence, and greater mating success. Subsequent research has amply confirmed that leaders in small-scale societies possess both characteristics. Neither Neel nor later theorists, however, adequately explained why knowledgeable, intelligent leaders are attractive both to followers and to mates. Building on the work of many others, we filled this gap by operationalizing leaders as individuals who regularly make decisions that benefit most members of the group. Good decision-making skills provided large benefits to mates and the family, with whom one cooperated for perhaps one's entire adult life, as well as to members of one's subsistence group and larger security and political groups. We further argued that decision-making that benefits others as well as oneself can be especially computationally complex, and therefore that sexual selection and biological market forces favoring these skills favored increased brain size. Finally, because parents must make decisions for their cognitively immature offspring, good decision-making abilities might have initially undergone strong selection in mothers, who provide most of the childcare in natural fertility populations.

Decision-making that benefits others is one example of a valuable computational service. Other important examples include threat and opportunity detection, gossip and information sharing, cultural transmission, story telling, medicinal knowledge, and advice and counsel. Providing computational services in exchange

for a variety of benefits would have helped subsidize a large, energetically expensive brain. Individuals who provided particularly valuable services gained prestige, i.e., additional benefits from fellow group members.

\section{References}

Alexander, G. E. \& Crutcher, M. D. (1990). Functional architecture of basal ganglia circuits: neural substrates of parallel processing. Trends in Neurosciences, 13(7), 266-271.

Alexander, R. D. (1974). The evolution of social behavior. Annual Review of Ecology and Systematics, (pp. $325-383)$.

Alexander, R. D. (1990). How did humans evolve? Reflections on the uniquely unique species. Museum of Zoology, The University of Michigan Special Publication, 1, 1-38.

Andersson, M. B. (1994). Sexual selection. Princeton University Press.

Antón, S. C., Potts, R., \& Aiello, L. C. (2014). Evolution of early Homo: an integrated biological perspective. Science, 345(6192), 1236828.

Archer, J. (2004). Sex differences in aggression in real-world settings: A meta-analytic review. Review of General Psychology, 8(4), 291.

Archer, J. \& Thanzami, V. (2009). The relation between mate value, entitlement, physical aggression, size and strength among a sample of young Indian men. Evolution and Human Behavior, 30(5), 315-321.

Arden, R., Gottfredson, L. S., \& Miller, G. (2009). Does a fitness factor contribute to the association between intelligence and health outcomes? Evidence from medical abnormality counts among 3654 US Veterans. Intelligence, 37(6), 581-591. 
Armbrust, M., Stoica, I., Zaharia, M., Fox, A., Griffith, R., Joseph, A. D., Katz, R., Konwinski, A., Lee, G., Patterson, D., \& Rabkin, A. (2010). A view of cloud computing. Communications of the ACM, 53(4), 50.

Ashton, B. J., Thornton, A., \& Ridley, A. R. (2018). An intraspecific appraisal of the social intelligence hypothesis. Philosophical Transactions of the Royal Society B: Biological Sciences, 373(1756), 20170288.

Axelrod, R. \& Hamilton, W. D. (1981). The Evolution of Cooperation. Science, 211(4489), 1390-1396.

Ball, G. F., Balthazart, J., \& McCarthy, M. M. (2014). Is it useful to view the brain as a secondary sexual characteristic? Neuroscience $\mathscr{E}$ Biobehavioral Reviews, 46, 628-638.

Barkow, J. H. (1980). Prestige and self-esteem: A biosocial interpretation. Dominance relations, (pp. 319-332).

Barkow, J. H. (1989). Darwin, sex, and status. University of Toronto Press.

Barkow, J. H., Akiwowo, A. A., Barua, T. K., Chance, M. R., Chapple, E. D., Chattopadhyay, G. P., Freedman, D. G., Geddes, W. R., Goswami, B. B., \& Isichei, P. A. C. (1975). Prestige and culture: a biosocial interpretation [and comments and replies]. Current Anthropology, (pp. 553-572).

Barton, R. A. \& Dunbar, R. I. (1997). Evolution of the social brain. In A. Whiten \& R. W. Byrne (Eds.), Machiavellian intelligence II: Extensions and evaluations, volume 2 (pp. 240-263).

Berdahl, A. M., Kao, A. B., Flack, A., Westley, P. A., Codling, E. A., Couzin, I. D., Dell, A. I., \& Biro, D. (2018). Collective animal navigation and migratory culture: From theoretical models to empirical evidence. Phil. Trans. R. Soc. B, 373(1746), 20170009.

Bickerton, D. \& Szathmáry, E. (2011). Confrontational scavenging as a possible source for language and cooperation. BMC Evolutionary Biology, 11(1), 261.

Binford, L. R. (2001). Constructing frames of reference: an analytical method for archaeological theory building using ethnographic and environmental data sets. University of California Press.

Bird, D. W., Bliege-Bird, R., Codding, B. F., \& Zeanah, D. W. (2019). Variability in the organization and size of hunter-gatherer groups: Foragers do not live in small-scale societies. Journal of Human Evolution, $131,96-108$.

Birdsell, J. B. (1958). On population structure in generalized hunting and collecting populations. Evolution, 12(2), 189-205.

Blurton Jones, N. G., Marlowe, F., Hawkes, K., \& O'Connell, J. F. (2000). Hunter-gatherer divorce rates and the paternal provisioning theory of human monogamy. In Adaptation and human behavior: an anthropological perspective. New York: Aldine de Gruyter. p (pp. 65-84).

Boehm, C. (1993). Egalitarian behavior and reverse dominance hierarchy. Current Anthropology, 34(3), 227.

Bogin, B., Bragg, J., \& Kuzawa, C. (2014). Humans are not cooperative breeders but practice biocultural reproduction. Annals of Human Biology, 41(4), 368-380.

Bolhuis, J. J., Tattersall, I., Chomsky, N., \& Berwick, R. C. (2014). How could language have evolved? PLoS Biology, 12(8), e1001934.

Bossaerts, P. \& Murawski, C. (2017). Computational complexity and human decision-making. Trends in Cognitive Sciences, 21, 917-929.

Bossaerts, P., Yadav, N., \& Murawski, C. (2019). Uncertainty and computational complexity. Philosophical Transactions of the Royal Society B: Biological Sciences, 374(1766), 20180138.

Bowles, S. (2009). Did warfare among ancestral hunter-gatherers affect the evolution of human social behaviors? Science, 324(5932), 1293-1298. 
Boyd, R. . \& Richerson, P. (1985). Culture and the Evolutionary Process. Chicago: University of Chicago Press.

Boydston, E. E., Morelli, T. L., \& Holekamp, K. E. (2001). Sex Differences in Territorial Behavior Exhibited by the Spotted Hyena (Hyaenidae, Crocuta crocuta). Ethology, 107(5), 369-385.

Brantingham, P. J. (1998). Hominid-carnivore coevolution and invasion of the predatory guild. Journal of Anthropological Archaeology, 17, 327-353.

Brent, L. J., Franks, D. W., Foster, E. A., Balcomb, K. C., Cant, M. A., \& Croft, D. P. (2015). Ecological knowledge, leadership, and the evolution of menopause in killer whales. Current Biology, 25(6), 746-750.

Brown, D. E. (1991). Human universals. McGraw-Hill New York.

Burkart, J. M., Hrdy, S. B., \& Van Schaik, C. P. (2009). Cooperative breeding and human cognitive evolution. Evolutionary Anthropology: Issues, News, and Reviews, 18(5), 175-186.

Buss, D. M. \& Schmitt, D. P. (2019). Mate preferences and their behavioral manifestations. Annual Review of Psychology, 70.

Byrne, R. \& Whiten, A. (1988). Machiavellian intelligence: social expertise and the evolution of intellect in monkeys, apes, and humans. Oxford University Press, USA.

Bøggild, T. \& Petersen, M. B. (2016). The evolved functions of procedural fairness: An adaptation for politics. In T. K. Shackelford \& R. D. Hansen (Eds.), The evolution of morality (pp. 247-276). Springer.

Carruthers, P. (2002). The cognitive functions of language. Behavioral and Brain Sciences, 25(6), 657-674.

Cavalli-Sforza, L. L. \& Feldman, M. W. (1981). Cultural transmission and evolution: A quantitative approach. Princeton, NJ: Princeton University Press.

Cavalli-Sforza, L. L., Feldman, M. W., Chen, K. H., \& Dornbusch, S. M. (1982). Theory and observation in cultural transmission. Science, 218, 19-27.

Cavazotte, F., Moreno, V., \& Hickmann, M. (2012). Effects of leader intelligence, personality and emotional intelligence on transformational leadership and managerial performance. The Leadership Quarterly, 23(3), 443-455.

Chagnon, N. A. (1988). Life histories, blood revenge, and warfare in a tribal population. Science, 239(4843), 985-92.

Chance, M. R. (1967). Attention structure as the basis of primate rank orders. Man, 2(4), 503-518.

Chapais, B. (2015). Competence and the evolutionary origins of status and power in humans. Hum Nature, $26(2), 161-83$.

Chapman, T., Arnqvist, G., Bangham, J., \& Rowe, L. (2003). Sexual conflict. Trends in Ecology EJ Evolution, 18(1), 41-47.

Cheng, J. T., Tracy, J. L., Foulsham, T., Kingstone, A., \& Henrich, J. (2013). Two ways to the top: evidence that dominance and prestige are distinct yet viable avenues to social rank and influence. J Pers Soc Psychol, 104(1), 103-25.

Cheng, J. T., Tracy, J. L., \& Henrich, J. (2010). Pride, personality, and the evolutionary foundations of human social status. Evolution and Human Behavior, 31(5), 334-347.

Choi, J.-K. \& Bowles, S. (2007). The coevolution of parochial altruism and war. science, 318(5850), 636-640.

Connelly, M. S., Gilbert, J. A., Zaccaro, S. J., Threlfall, K. V., Marks, M. A., \& Mumford, M. D. (2000). Exploring the relationship of leadership skills and knowledge to leader performance. The Leadership Quarterly, 11(1), 65-86. 
Connor, R. (1986). Pseudo-reciprocity: Investing in mutualism. Animal Behaviour, 34, 1562-1584.

Connor, R. C. (1995). Altruism among non-relatives: alternatives to the 'Prisoner's Dilemma'. Trends in Ecology $\&$ Evolution, 10(2), 84-86.

Conroy-Beam, D., Buss, D. M., Pham, M. N., \& Shackelford, T. K. (2015). How sexually dimorphic are human mate preferences? Personality and Social Psychology Bulletin, 41(8), 1082-1093.

Corballis, M. C. (2017). Language evolution: A changing perspective. Trends in Cognitive Sciences, 21(4), 229-236.

Couzin, I. D. \& Krause, J. (2003). Self-organization and collective behavior in vertebrates. Advances in the Study of Behavior, 32, 1-75.

Couzin, I. D., Krause, J., Franks, N. R., \& Levin, S. A. (2005). Effective leadership and decision-making in animal groups on the move. Nature, 433(7025), 513-516.

Cowlishaw, G. \& Dunbar, R. I. (1991). Dominance rank and mating success in male primates. Animal Behaviour, 41(6), 1045-1056.

Cox, S., Ritchie, S., Fawns-Ritchie, C., Tucker-Drob, E., \& Deary, I. (2019). Brain imaging correlates of general intelligence in uk biobank. bioRxiv.

da Cruz, J., Rodrigues, J., Thoresen, J. C., Chicherov, V., Figueiredo, P., Herzog, M. H., \& Sandi, C. (2018). Dominant men are faster in decision-making situations and exhibit a distinct neural signal for promptness. Cerebral Cortex, 28(10), 3740-3751.

Darwin, C. (1871). The descent of man and selection in relation to sex. London: John Murray.

Dean, W. (2016). Computational complexity theory. In E. N. Zalta (Ed.), The Stanford Encyclopedia of Philosophy. Metaphysics Research Lab, Stanford University, winter 2016 edition.

DeCasien, A. R., Williams, S. A., \& Higham, J. P. (2017). Primate brain size is predicted by diet but not sociality. Nature Ecology \& Evolution, 1(5).

DeVore, I. \& Washburn, S. L. (1963). Baboon ecology and human evolution. In C. Howell \& F. Bourliere (Eds.), African ecology and human evolution (pp. 335-367). Aldine, Chicago.

Dominguez-Rodrigo, M. \& Pickering, T. R. (2017). The meat of the matter: an evolutionary perspective on human carnivory. Azania: Archaeological Research in Africa, 52(1), 4-32.

Drews, C. (1993). The concept and definition of dominance in animal behaviour. Behaviour, 125(3), 283-313.

Dyble, M., Salali, G. D., Chaudhary, N., Page, A., Smith, D., Thompson, J., Vinicius, L., Mace, R., \& Migliano, A. B. (2015). Sex equality can explain the unique social structure of hunter-gatherer bands. Science, 348(6236), 796-798.

Ekman, P. (2016). What scientists who study emotion agree about. Perspectives on Psychological Science, 11(1), 31-34.

Engle, P. L. (1991). Maternal work and child-care strategies in peri-urban Guatemala: Nutritional effects. Child development, 62(5), 954-965.

Everaert, M. B., Huybregts, M. A., Berwick, R. C., Chomsky, N., Tattersall, I., Moro, A., \& Bolhuis, J. J. (2017). What is language and how could it have evolved? Trends in Cognitive Sciences, 21(8), 569-571.

Ferraro, J. V., Plummer, T. W., Pobiner, B. L., Oliver, J. S., Bishop, L. C., Braun, D. R., Ditchfield, P. W., Seaman III, J. W., Binetti, K. M., Seaman Jr, J. W., et al. (2013). Earliest archaeological evidence of persistent hominin carnivory. PLOS ONE, 8(4), e62174.

Flinn, M. V., Geary, D. C., \& Ward, C. V. (2005). Ecological dominance, social competition, and coalitionary arms races. Evolution and Human Behavior, 26(1), 10-46. 
Fossey, D. (1972). Vocalizations of the mountain Gorilla (Gorilla gorilla beringei). Animal Behaviour, 20(1), $36-53$.

Foulsham, T., Cheng, J. T., Tracy, J. L., Henrich, J., \& Kingstone, A. (2010). Gaze allocation in a dynamic situation: Effects of social status and speaking. Cognition, 117(3), 319-331.

Fried, M. H. (1967). The evolution of political society. Random House.

Furuichi, T. (2011). Female contributions to the peaceful nature of bonobo society. Evolutionary Anthropology: Issues, News, and Reviews, 20(4), 131-142.

Garfield, Z. H., Garfield, M. J., \& Hewlett, B. S. (2016). A Cross-Cultural Analysis of Hunter-Gatherer Social Learning. In B. S. Hewlett \& T. Hideaki (Eds.), Social Learning and Innovation in Contemporary Hunter-Gatherers (pp. 19-34). Springer.

Garfield, Z. H. \& Hagen, E. H. (2019). Investigating evolutionary models of leadership among recently settled Ethiopian hunter-gatherers. The Leadership Quarterly, Forthcoming.

Garfield, Z. H., Hubbard, Robert, H., \& Hagen, E. H. (2019). Evolutionary models of leadership: Tests and synthesis. Human Nature.

Garfield, Z. H., von Rueden, C., \& Hagen, E. H. (2018). The evolutionary anthropology of political leadership. The Leadership Quarterly.

Garland, J., Berdahl, A. M., Sun, J., \& Bollt, E. (2018). The anatomy of leadership in collective behaviour. arXiv preprint arXiv:1802.01194.

Gaulin, S. J. \& FitzGerald, R. W. (1986). Sex differences in spatial ability: an evolutionary hypothesis and test. The American Naturalist, 127(1), 74-88.

Gavrilets, S. (2015). Collective action problem in heterogeneous groups. Phil. Trans. R. Soc. B, 370, 20150016 .

Gavrilets, S. \& Fortunato, L. (2014). A solution to the collective action problem in between-group conflict with within-group inequality. Nature communications, 5.

Geary, D. C. \& Flinn, M. V. (2001). Evolution of human parental behavior and the human family. Parenting, $1(1-2), 5-61$.

Gigerenzer, G. \& Selten, R. (2002). Bounded rationality: The adaptive toolbox. MIT press.

Gigerenzer, G. \& Todd, P. M. (1999). Simple heuristics that make us smart. Oxford University Press.

Glowacki, L. \& von Rueden, C. R. (2015). Leadership solves collective action problems in small-scale societies. Philos Trans R Soc Lond B Biol Sci, 370(1683).

Glowacki, L., Wilson, M. L., \& Wrangham, R. W. (2017). The evolutionary anthropology of war. Journal of Economic Behavior and Organization.

Glowacki, L. \& Wrangham, R. W. (2013). The role of rewards in motivating participation in simple warfare. Hum Nat, 24(4), 444-60.

Goldstein, D. G. \& Gigerenzer, G. (2011). Models of ecological rationality: The recognition heuristic. In Heuristics (pp. 60-80). Oxford University Press.

Gonzalez-Forero M \& Gardner A (2018). Inference of ecological and social drivers of human brain-size evolution. Nature, 557(7706), 554-557. OCLC: 7671188160.

Gurven, M., Allen-Arave, W., Hill, K., \& Hurtado, M. (2000a). "It's a wonderful life": signaling generosity among the Ache of Paraguay. Evolution and Human Behavior, 21(4), 263-282. 
Gurven, M., Allen-Arave, W., Hill, K., \& Hurtado, M. (2000b). "it's a wonderful life": signaling generosity among the Ache of Paraguay. Evolution and Human Behavior, 21(4), 263-282.

Gurven, M., Hill, K., Hames, R., Kameda, T., McDermott, R., Lupo, K., Kiahtipes, C., Ragir, S., Rosas, A., Gurven, M., et al. (2009). Why do men hunt? a reevaluation of "man the hunter" and the sexual division of labor. Current Anthropology, 50(1), 51-74.

Guttal, V. \& Couzin, I. D. (2011). Leadership, collective motion and the evolution of migratory strategies. Communicative $\mathcal{E}$ Integrative Biology, 4(3), 294-298.

Hames, R. (2015). Kin selection. In D. Buss (Ed.), The Handbook of Evolutionary Psychology, volume 2 (pp. $505-523)$.

Hamilton, M. J., Milne, B. T., Walker, R. S., Burger, O., \& Brown, J. H. (2007). The complex structure of hunter-gatherer social networks. Proceedings of the Royal Society B: Biological Sciences, 274(1622), 2195-2203.

Hammerstein, P., Hagen, E. H., \& Laubichler, M. D. (2006). The strategic view of biological agents. Biological Theory, 1(2), 191-194.

Hammerstein, P. \& Noë, R. (2016). Biological trade and markets. Phil. Trans. R. Soc. B, 371, 20150101.

Harcourt, A. H. \& De Waal, F. B. (1992). Coalitions and alliances in humans and other animals. Oxford University Press.

Hawley, P. H. (1999). The ontogenesis of social dominance: A strategy-based evolutionary perspective. Developmental Review, 19(1), 97-132.

Henrich, J., Chudek, M., \& Boyd, R. (2015). The Big Man Mechanism: how prestige fosters cooperation and creates prosocial leaders. Phil. Trans. R. Soc. B, 370(1683), 20150013.

Henrich, J. \& Gil-White, F. J. (2001). The evolution of prestige: freely conferred deference as a mechanism for enhancing the benefits of cultural transmission. Evolution and Human Behavior, 22(3), 165-196.

Henrich, J. \& Henrich, N. (2010). The evolution of cultural adaptations: Fijian food taboos protect against dangerous marine toxins. Proceedings of the Royal Society of London B: Biological Sciences, 277(1701), $3715-3724$.

Henrich, N. \& Henrich, J. P. (2007). Why humans cooperate: A cultural and evolutionary explanation. Oxford University Press.

Henschel, J. R. \& Skinner, J. D. (1991). Territorial Behaviour by a Clan of Spotted Hyaenas Crocuta crocuta. Ethology, 88(3), 223-235.

Herculano-Houzel, S. (2012). The remarkable, yet not extraordinary, human brain as a scaled-up primate brain and its associated cost. Proceedings of the National Academy of Sciences, 109(Supplement_1), 1066110668.

Hess, N. H. \& Hagen, E. H. (2006). Sex differences in indirect aggression: Psychological evidence from young adults. Evolution and Human Behavior, 27(3), 231-245.

Hess, N. H. \& Hagen, E. H. (2017). Gossip, reputation, and friendship in within-group competition: an evolutionary perspective. In Handbook of Reputation and Gossip. Oxford University Press.

Hewlett, B. S. (1991). Demography and Childcare in Preindustrial Societies. Journal of Anthropological Research, 47, 1-37.

Hewlett, B. S. (1993). Intimate fathers: The nature and context of Aka Pygmy paternal infant care. University of Michigan Press. 
Heyer, E., Chaix, R., Pavard, S., \& Austerlitz, F. (2012). Sex-specific demographic behaviours that shape human genomic variation. Molecular ecology, 21(3), 597-612.

Hill, K. R., Walker, R. S., Bo\vzi\vcević, M., Eder, J., Headland, T., Hewlett, B., Hurtado, A. M., Marlowe, F., Wiessner, P., \& Wood, B. (2011). Co-residence patterns in hunter-gatherer societies show unique human social structure. Science, 331(6022), 1286-1289.

Hill, K. R., Wood, B. M., Baggio, J., Hurtado, A. M., \& Boyd, R. T. (2014). Hunter-gatherer inter-band interaction rates: Implications for cumulative culture. PLoS One, 9(7), e102806.

Hills, T. T., Todd, P. M., Lazer, D., Redish, A. D., Couzin, I. D., Group, C. S. R., et al. (2015). Exploration versus exploitation in space, mind, and society. Trends in Cognitive Sciences, 19(1), 46-54.

Hooper, P. L., Kaplan, H. S., \& Boone, J. L. (2010). A theory of leadership in human cooperative groups. Journal of Theoretical Biology, 265(4), 633-646.

Horner, V., Proctor, D., Bonnie, K. E., Whiten, A., \& Waal, F. B. M. d. (2010). Prestige affects cultural learning in chimpanzees. PLOS ONE, 5(5), e10625.

Hrdy, S. B. (2006). Evolutionary context of human development. The cooperative breeding model. Family Relationships, (pp.30).

Hrdy, S. B. (2011). Mothers and others. Harvard University Press.

Humphrey, L. T. (2010). Weaning behaviour in human evolution. Seminars in Cell $\mathscr{E}$ Developmental Biology, 21(4), 453-461.

Humphrey, N. K. (1976). The social function of intellect. In P. D. Bateson \& R. L. Hinde (Eds.), Growing points in ethology (pp. 303-317). Cambridge: Cambridge University Press.

Irons, W. (1998). Adaptively Relevant Environments versus the Environment of Evolutionary Adaptedness. Evolutionary Anthropology, 6, 194-204.

Isler Karin \& van Schaik Carel P (2006). Metabolic costs of brain size evolution. Biology Letters, 2(4), $557-560$.

Jiménez, Á. V. \& Mesoudi, A. (2019). Prestige-biased social learning: current evidence and outstanding questions. Palgrave Communications, 5(1), 20.

Jobling, M. A. \& Tyler-Smith, C. (2017). Human Y-chromosome variation in the genome-sequencing era. Nature Reviews Genetics, 18(8), 485-497. OCLC: 7049359785.

Johnson, D. D. P. (2015). Leadership in War: Evolution, Cognition, and the Military Intelligence Hypothesis. In The Handbook of Evolutionary Psychology (pp. 1-23). American Cancer Society.

Jonason, P. K., Marsh, K., Dib, O., Plush, D., Doszpot, M., Fung, E., Crimmins, K., Drapski, M., \& Di Pietro, K. (2019). Is smart sexy? Examining the role of relative intelligence in mate preferences. Personality and Individual Differences, 139, 53-59.

Jones, A. G. \& Ratterman, N. L. (2009). Mate choice and sexual selection: what have we learned since Darwin? Proceedings of the National Academy of Sciences, 106(Supplement 1), 10001-10008.

Judge, T. A., Colbert, A. E., \& Ilies, R. (2004). Intelligence and leadership: a quantitative review and test of theoretical propositions. J Appl Psychol, 89(3), 542-52.

Kantner, J. (2010). Identifying the pathways to permanent leadership. In K. J. Vaughn, J. W. Eerkens, \& J. Kanter (Eds.), The Evolution of Leadership: Transitions in Decision Making from Small-Scale to Middle-Range Societies (pp. 249-281). Santa Fe: SAR.

Kaplan, H., Hill, K., Lancaster, J., \& Hurtado, A. M. (2000). A theory of human life history evolution: Diet, intelligence, and longevity. Evolutionary Anthropology, 9(4), 156-185. 
Kaplan, H., Mueller, T., Gangestad, S., \& Lancaster, J. (2003). Neural capital and life span evolution among primates and humans. In C. Finch, J. Robine, \& Y. Christen (Eds.), Brain and longevity (pp. 69-97). Springer.

Kappeler, P. M. \& Van Schaik, C. P. (2004). Sexual selection in primates: new and comparative perspectives. Cambridge University Press.

Karmin, M., Saag, L., Vicente, M., Sayres, M. A. W., Järve, M., Talas, U. G., Rootsi, S., Ilumäe, A.-M., Mägi, R., Mitt, M., Pagani, L., Puurand, T., Faltyskova, Z., Clemente, F., Cardona, A., Metspalu, E., Sahakyan, H., Yunusbayev, B., Hudjashov, G., DeGiorgio, M., Loogväli, E.-L., Eichstaedt, C., Eelmets, M., Chaubey, G., Tambets, K., Litvinov, S., Mormina, M., Xue, Y., Ayub, Q., Zoraqi, G., Korneliussen, T. S., Akhatova, F., Lachance, J., Tishkoff, S., Momynaliev, K., Ricaut, F.-X., Kusuma, P., Razafindrazaka, H., Pierron, D., Cox, M. P., Sultana, G. N. N., Willerslev, R., Muller, C., Westaway, M., Lambert, D., Skaro, V., Kovačevic', L., Turdikulova, S., Dalimova, D., Khusainova, R., Trofimova, N., Akhmetova, V., Khidiyatova, I., Lichman, D. V., Isakova, J., Pocheshkhova, E., Sabitov, Z., Barashkov, N. A., Nymadawa, P., Mihailov, E., Seng, J. W. T., Evseeva, I., Migliano, A. B., Abdullah, S., Andriadze, G., Primorac, D., Atramentova, L., Utevska, O., Yepiskoposyan, L., Marjanovic', D., Kushniarevich, A., Behar, D. M., Gilissen, C., Vissers, L., Veltman, J. A., Balanovska, E., Derenko, M., Malyarchuk, B., Metspalu, A., Fedorova, S., Eriksson, A., Manica, A., Mendez, F. L., Karafet, T. M., Veeramah, K. R., Bradman, N., Hammer, M. F., Osipova, L. P., Balanovsky, O., Khusnutdinova, E. K., Johnsen, K., Remm, M., Thomas, M. G., Tyler-Smith, C., Underhill, P. A., Willerslev, E., Nielsen, R., Metspalu, M., Villems, R., \& Kivisild, T. (2015). A recent bottleneck of $\mathrm{Y}$ chromosome diversity coincides with a global change in culture. Genome Research, 25(4), 459-466.

Kelly, R. L. (1983). Hunter-gatherer mobility strategies. Journal of Anthropological Research, 39(3), 277-306.

Kendal, R., Hopper, L. M., Whiten, A., Brosnan, S. F., Lambeth, S. P., Schapiro, S. J., \& Hoppitt, W. (2015). Chimpanzees copy dominant and knowledgeable individuals: Implications for cultural diversity. Evolution and Human Behavior, 36(1), 65-72.

Kokko, H. (2003). Are reproductive skew models evolutionarily stable? Proceedings of the Royal Society of London B: Biological Sciences, 270(1512), 265-270.

Koykka, C. \& Wild, G. (2015). The evolution of group dispersal with leaders and followers. Journal of Theoretical Biology, 371, 117-126.

Kramer, K. L. (2010). Cooperative breeding and its significance to the demographic success of humans. Annual Review of Anthropology, 39, 417-436.

Lamm, C., Batson, C. D., \& Decety, J. (2007). The neural substrate of human empathy: effects of perspectivetaking and cognitive appraisal. Journal of Cognitive Neuroscience, 19(1), 42-58.

Lebel, C. \& Beaulieu, C. (2011). Longitudinal Development of Human Brain Wiring Continues from Childhood into Adulthood. Journal of Neuroscience, 31(30), 10937-10947.

Leca, J.-B. . B., Gunst, N., Thierry, B., \& Petit, O. (2003). Distributed leadership in semifree-ranging white-faced capuchin monkeys. Animal Behaviour, 66(6), 1045-1052.

Lee, A. J., Dubbs, S. L., Von Hippel, W., Brooks, R. C., \& Zietsch, B. P. (2014). A multivariate approach to human mate preferences. Evolution and Human Behavior, 35(3), 193-203.

Leimar, O. \& Connor, R. C. (2003). By-product benefits, reciprocity, and pseudoreciprocity in mutualism. In P. Hammerstein (Ed.), Genetic and Cultural Evolution of Cooperation (pp. 203-222). MIT Press Cambridge, MA.

Leimar, O. \& Hammerstein, P. (2010). Cooperation for direct fitness benefits. Philosophical Transactions of the Royal Society B: Biological Sciences, 365(1553), 2619-2626. 
Levine, S. C., Foley, A., Lourenco, S., Ehrlich, S., \& Ratliff, K. (2016). Sex differences in spatial cognition: Advancing the conversation. Wiley Interdisciplinary Reviews: Cognitive Science, 7(2), 127-155.

Lieder, F. \& Griffiths, T. L. (2019). Resource-rational analysis: understanding human cognition as the optimal use of limited computational resources. Behavioral and Brain Sciences, (pp. 1-85).

Lippold, S., Xu, H., Ko, A., Li, M., Renaud, G., Butthof, A., Schröder, R., \& Stoneking, M. (2014). Human paternal and maternal demographic histories: insights from high-resolution Y chromosome and mtDNA sequences. Investigative genetics, 5(1), 1.

Lopez, A. C. (2016). The evolution of war: theory and controversy. Int Theory International Theory, 8, $97-139$.

Macfarlan, S. J., Remiker, M., \& Quinlan, R. (2012). Competitive altruism explains labor exchange variation in a Dominican community. Current Anthropology, 53, 118-124.

MacLean, E. L., Hare, B., Nunn, C. L., Addessi, E., Amici, F., Anderson, R. C., Aureli, F., Baker, J. M., Bania, A. E., Barnard, A. M., Boogert, N. J., Brannon, E. M., Bray, E. E., Bray, J., Brent, L. J. N., Burkart, J. M., Call, J., Cantlon, J. F., Cheke, L. G., Clayton, N. S., Delgado, M. M., DiVincenti, L. J., Fujita, K., Herrmann, E., Hiramatsu, C., Jacobs, L. F., Jordan, K. E., Laude, J. R., Leimgruber, K. L., Messer, E. J. E., de A. Moura, A. C., Ostojić, L., Picard, A., Platt, M. L., Plotnik, J. M., Range, F., Reader, S. M., Reddy, R. B., Sandel, A. A., Santos, L. R., Schumann, K., Seed, A. M., Sewall, K. B., Shaw, R. C., Slocombe, K. E., Su, Y., Takimoto, A., Tan, J., Tao, R., van Schaik, C. P., Virányi, Z., Visalberghi, E., Wade, J. C., Watanabe, A., Widness, J., Young, J. K., Zentall, T. R., \& Zhao, Y. (2014). The evolution of self-control. Proceedings of the National Academy of Sciences, 111(20), E2140-E2148.

Maner, J. K., DeWall, C. N., \& Gailliot, M. T. (2008). Selective attention to signs of success: Social dominance and early stage interpersonal perception. Personality and Social Psychology Bulletin, 34(4), $488-501$.

Manuel, D.-R. \& Rayne, P. T. (2003). Early hominid hunting and scavenging: A zooarcheological review. Evolutionary Anthropology: Issues, News, and Reviews, 12(6), 275-282.

Marlowe, F. W. (2004). Mate preferences among Hadza hunter-gatherers. Human Nature, 15(4), 365-376.

Marlowe, F. W. (2005). Hunter-gatherers and human evolution. Evolutionary Anthropology, 14(2), 54-67.

Martin, A. \& Santos, L. R. (2016). What cognitive representations support primate theory of mind? Trends in Cognitive Sciences, 20(5), 375-382.

Maynard Smith, J. \& Parker, G. A. (1976). The logic of asymmetric contests. Animal behaviour, 24(1), $159-175$.

Meehan, C. L., Helfrecht, C., \& Malcom, C. D. (2016). Implications of Lengthy Development and Maternal Life History. In Childhood: Origins, Evolution, and Implications (pp. 199). University of New Mexico Press.

Mell, P. \& Grance, T. (2011). The NIST definition of cloud computing.

Menzel, E.W., J. (1971). Communication about the environment in a group of young chimpanzees. Folia Primatologica, 15(3-4), 220-232.

Miller, G. (2000a). The mating mind: How sexual choice shaped the evolution of human nature. Doubleday Books.

Miller, G. (2000b). Sexual selection for indicators of intelligence. In Novartis Foundation Symposium (pp. 260-270).: Chichester; New York; John Wiley; 1999.

Miller, G. F. (1998). How mate choice shaped human nature: A review of sexual selection and human evolution. Handbook of evolutionary psychology: Ideas, issues, and applications, (pp. 87-129). 
Milton, K. (1988). Foraging behaviour and the evolution of primate intelligence. Clarendon Press/Oxford University Press.

Miner, E. J., Gurven, M., Kaplan, H., \& Gaulin, S. J. (2014). Sex difference in travel is concentrated in adolescence and tracks reproductive interests. Proceedings of the Royal Society of London B: Biological Sciences, 281(1796), 20141476.

Mock, D. W. \& Parker, G. A. (1997). The evolution of sibling rivalry. oxford series in ecology and evolution.

Mueller, T., O'Hara, R. B., Converse, S. J., Urbanek, R. P., \& Fagan, W. F. (2013). Social learning of migratory performance. Science, 341(6149), 999-1002.

Murdock, G. P. (1949). Social structure.

Muthukrishna, M., Doebeli, M., Chudek, M., \& Henrich, J. (2018). The cultural brain hypothesis: How culture drives brain expansion, sociality, and life history. PLOS Computational Biology, 14(11), e1006504.

Navarrete, A., van Schaik, C. P., \& Isler, K. (2011). Energetics and the evolution of human brain size. Nature, 480(7375), 91-93.

Nave, G., Jung, W. H., Linnér, R. K., Kable, J. W., \& Koellinger, P. D. (2019). Are bigger brains smarter? evidence from a large-scale preregistered study. Psychological Science, 0(0), 0956797618808470.

Neel, J. V. (1980). On being headman. Perspectives in Biology and Medicine, 23(277-94).

Neel, J. V. \& Salzano, F. M. (1967). Further studies on the Xavante Indians. X. Some hypothesesgeneralizations resulting from these studies. American Journal of Human Genetics, 19, 554.

Neel, J. V., Salzano, F. M., Junqueira, P. C., Keiter, F., \& Maybury-Lewis, D. (1964). Studies on the Xavante Indians of the Brazilian Mato Grosso. American Journal of Human Genetics, 16, 52-140.

Nelson, M. E. \& Bower, J. M. (1990). Brain maps and parallel computers. Trends in Neurosciences, 13(10), 403-408.

Noë, R. \& Hammerstein, P. (1995). Biological markets. Trends in Ecology \& Evolution, 10(8), 336-339.

Oliveira, S., Hübner, A., Fehn, A.-M., Aço, T., Lages, F., Pakendorf, B., Stoneking, M., \& Rocha, J. (2018). The role of matrilineality in shaping patterns of $Y$ chromosome and mtDNA sequence variation in southwestern Angola. bioRxiv, (pp. 349878).

Olson, M. (1965). Logic of collective action: Public goods and the theory of groups. Cambridge, MA.

Parker, G. A., Royle, N. J., \& Hartley, I. R. (2002). Intrafamilial conflict and parental investment: a synthesis. Philosophical Transactions of the Royal Society B: Biological Sciences, 357(1419), 295-307.

Payne, K. (2003). Sources of social complexity in the three elephant species. In F. B. De Waal \& P. L. Tyack (Eds.), Animal social complexity: Intelligence, culture, and individualized societies (pp. 57-85). Cambridge, MA, US: Harvard University Press.

Pereira, A. G. \& Moita, M. A. (2016). Is there anybody out there? Neural circuits of threat detection in vertebrates. Current opinion in neurobiology, 41, 179-187.

Petersen, M. B., Sell, A., Tooby, J., \& Cosmides, L. (2010). Evolutionary Psychology and Criminal Justice: A Recalibrational Theory of Punishment and Reconciliation. In H. Høegh-Olesen (Ed.), Human morality 83 sociality: Evolutionary 83 comparative perspectives (pp. 72-131). New York: Palgrave Macmillan.

Peterson, M., Warf, B. C., \& Schiff, S. J. (2018). Normative human brain volume growth. Journal of Neurosurgery: Pediatrics, 21(5), 478-485.

Pheasant, S. T. (1983). Sex differences in strength-some observations on their variability. Applied Ergonomics, 14(3), 205-211. 
Piantadosi, S. T. \& Kidd, C. (2016). Extraordinary intelligence and the care of infants. Proceedings of the National Academy of Sciences, 113(25), 6874-6879.

Pietschnig, J., Penke, L., Wicherts, J. M., Zeiler, M., \& Voracek, M. (2015). Meta-analysis of associations between human brain volume and intelligence differences: How strong are they and what do they mean? Neuroscience $\& 3$ Biobehavioral Reviews, 57, 411-432.

Pinker, S. \& Jackendoff, R. (2005). The faculty of language: what's special about it? Cognition, 95(2), 201-236.

Placek, C. D., Madhivanan, P., \& Hagen, E. H. (2017). Innate food aversions and culturally transmitted food taboos in pregnant women in rural southwest india: Separate systems to protect the fetus? Evolution and Human Behavior, 38(6), 714-728.

Plavcan, J. M. (2012a). Body size, size variation, and sexual size dimorphism in early Homo. Current Anthropology, 53(S6), S409-S423.

Plavcan, J. M. (2012b). Sexual size dimorphism, canine dimorphism, and male-male competition in primates. Human Nature, 23(1), 45-67.

Powell, L. E., Isler, K., \& Barton, R. A. (2017). Re-evaluating the link between brain size and behavioural ecology in primates. Proceedings of the Royal Society B: Biological Sciences, 284(1865), 20171765.

Premack, D. \& Woodruff, G. (1978). Does the chimpanzee have a theory of mind? Behavioral and Brain Sciences, 1(4), 515-526.

Price, M. E. \& Van Vugt, M. (2014). The evolution of leader-follower reciprocity: the theory of service-forprestige. Frontiers in Human Neuroscience, 8(363), 1-17.

Price, M. E. \& Van Vugt, M. (2015). The Service-for-Prestige Theory of Leader-Follower Relations: A Review of the Evolutionary Psychology and Anthropology Literatures. In R. D. Arvey \& S. M. Colarelli (Eds.), Biological Foundations of Organizational Behavior (pp. 397-477). Chicago: The University of Chicago Press.

Price, M. E., Van Vugt, M., Arvey, R. D., \& Colarelli, S. M. (2014). The Service-for-Prestige Theory of Leader-Follower Relations: A Review of the Evolutionary Psychology and Anthropology Literatures. Biological Foundations of Organizational Behavior.

Price, T., Wadewitz, P., Cheney, D., Seyfarth, R., Hammerschmidt, K., \& Fischer, J. (2015). Vervets revisited: A quantitative analysis of alarm call structure and context specificity. Scientific reports, 5, 13220.

Prokosch, M. D., Coss, R. G., Scheib, J. E., \& Blozis, S. A. (2009). Intelligence and mate choice: intelligent men are always appealing. Evolution and Human Behavior, 30(1), 11-20.

Puts, D. (2016). Human sexual selection. Current opinion in psychology, 7, 28-32.

Queller, D. C. (2011). Expanded social fitness and hamilton's rule for kin, kith, and kind. Proceedings of the National Academy of Sciences, 108(Supplement 2), 10792-10799.

Queller, D. C. \& Strassmann, J. E. (2018). Evolutionary conflict. Annual Review of Ecology, Evolution, and Systematics, 49, 73-93.

Rice, W. R. (1984). Sex chromosomes and the evolution of sexual dimorphism. Evolution, 38(4), 735.

Richerson, P. \& Henrich, J. (2012). Tribal Social Instincts and the Cultural Evolution of Institutions to Solve Collective Action Problems. Cliodynamics, 3(1).

Richerson, P. J. \& Boyd, R. (2000). Climate, culture and the evolution of cognition. In C. Heyes \& L. Huber (Eds.), The Evolution of Cognition (pp. 329-346). MIT Press. 
Richerson, P. J. \& Boyd, R. (2008). Not by genes alone: How culture transformed human evolution. University of Chicago press.

Roberts, G. (2005). Cooperation through interdependence. Animal Behaviour, 70(4), 901-908.

Rodseth, L., Wrangham, R. W., Harrigan, A. M., Smuts, B. B., Dare, R., Fox, R., King, B. J., Lee, P. C., Foley, R. A., \& Muller, J. C. (1991). The Human Community as a Primate Society [and Comments]. Current Anthropology, (pp. 221-254).

Rosati, A. G. (2017). Foraging cognition: Reviving the ecological intelligence hypothesis. Trends in Cognitive Sciences, 21(9), 691-702.

Roscoe, P. (2007). Intelligence, coalitional killing, and the antecedents of war. American Anthropologist, 109(3), 485-495.

Roscoe, P. (2009). Social Signaling and the Organization of Small-Scale Society: The Case of Contact-Era New Guinea. Journal of Archaeological Method and Theory, 16(2), 69-116.

Royle, N. J., Smiseth, P. T., \& Kölliker, M. (2012). The evolution of parental care. Oxford University Press.

Rubinstein, A. (1998). Modeling bounded rationality. MIT press.

Ruigrok, A. N., Salimi-Khorshidi, G., Lai, M.-C., Baron-Cohen, S., Lombardo, M. V., Tait, R. J., \& Suckling, J. (2014). A meta-analysis of sex differences in human brain structure. Neuroscience 8 Biobehavioral Reviews, 39, 34-50.

Ruttan, n. \& Borgerhoff Mulder M, n. (1999). Are East African Pastoralists Truly Conservationists? Current Anthropology, 40(5), 621-652.

Sapolsky, R. M. (2005). The influence of social hierarchy on primate health. Science, 308, 648-652.

Schaafsma, S. M., Pfaff, D. W., Spunt, R. P., \& Adolphs, R. (2015). Deconstructing and reconstructing theory of mind. Trends in Cognitive Sciences, 19(2), 65-72.

Schaller, G. B. \& Lowther, G. R. (1969). The relevance of carnivore behavior to the study of early hominids. Southwestern Journal of Anthropology, (pp. 307-341).

Schmitt, D. P. (2003). Universal sex differences in the desire for sexual variety: Tests from 52 nations, 6 continents, and 13 islands. Journal of Personality and Social Psychology, 85(1), 85.

Schmitt, D. P. (2005). Sociosexuality from Argentina to Zimbabwe: A 48-nation study of sex, culture, and strategies of human mating. Behavioral and Brain Sciences, 28(2), 247-275.

Schmitt, D. P. (2014). Evaluating Evidence of Mate Preference Adaptations: How Do We Really Know What Homo Sapiens Sapiens Really Want? In V. A. Weekes-Shackelford \& T. K. Shackelford (Eds.), Evolutionary Perspectives on Human Sexual Psychology and Behavior (pp. 3-39). New York, NY: Springer New York.

Schoenemann, P. T. (2013). Hominid brain evolution. In D. R. Begun (Ed.), A companion to paleoanthropology (pp. 136-164). Blackwell Publishing.

Selten, R. (1990). Bounded rationality. Journal of Institutional and Theoretical Economics (JITE)/Zeitschrift für die gesamte Staatswissenschaft, 146(4), 649-658.

Selten, R. (1998). Aspiration adaptation theory. Journal of Mathematical Psychology, 42(2-3), 191-214.

Service, E. R. (1964). Primitive social organization. Random House.

Seyfarth, R. M., Cheney, D. L., \& Marler, P. (1980). Vervet monkey alarm calls: semantic communication in a free-ranging primate. Animal Behaviour, 28(4), 1070-1094. 
Shen, S.-F. \& Reeve, H. K. (2010). Reproductive skew theory unified: The general bordered tug-of-war model. Journal of Theoretical Biology, 263, 1-12.

Sherman, P. W. (1977). Nepotism and the evolution of alarm calls. Science, 197(4310), 1246-1253.

Sherman, P. W. (1985). Alarm calls of Belding's ground squirrels to aerial predators: nepotism or selfpreservation? Behavioral Ecology and Sociobiology, 17(4), 313-323.

Silverman, I. \& Eals, M. (1992). Sex differences in spatial abilities: evolutionary theory and data. In J. Barkow, L. Cosmides, \& J. Tooby (Eds.), The Adapted Mind: Evolutionary Psychology and the Generation of Culture (pp. 487-503). New York: Oxford University Press.

Simon, H. A. (1955). A behavioral model of rational choice. The Quarterly Journal of Economics, 69(1), $99-118$

Simon, H. A. (1956). Rational choice and the structure of the environment. Psychological Review, 63(2), $129-138$.

Simon, H. A. (1972). Theories of bounded rationality. In C. B. McGuire \& R. Radner (Eds.), Decision and Organization, volume 1 (pp. 161-176). North-Holland.

Smith, E. A. \& Choi, J.-K. . K. (2007). The emergence of inequality in small-scale societies: simple scenarios and agent-based simulations. The model-based archaeology of socionatural systems, (pp. 105-20).

Smith, J. E., Gavrilets, S., Mulder, M. B., Hooper, P. L., Mouden, C. E., Nettle, D., Hauert, C., Hill, K., Perry, S., Pusey, A. E., van Vugt, M., \& Smith, E. A. (2016). Leadership in Mammalian Societies: Emergence, Distribution, Power, and Payoff. Trends Ecol Evol, 31(1), 54-66.

Smith, J. E., Ortiz, C. A., Buhbe, M. T., \& van Vugt, M. (2018). Obstacles and opportunities for female leadership in mammalian societies: A comparative perspective. The Leadership Quarterly.

Smith, J. E., Swanson, E. M., Reed, D., \& Holekamp, K. E. (2012). Evolution of cooperation among mammalian carnivores and its relevance to hominin evolution. Current Anthropology, 53(S6), S436-S452.

Starkweather, K. E. \& Keith, M. H. (2018). Estimating impacts of the nuclear family and heritability of nutritional outcomes in a boat-dwelling community. American Journal of Human Biology, (pp. n/a-n/a).

Stiner, M. C. (2002). Carnivory, coevolution, and the geographic spread of the genus Homo. Journal of Archaeological Research, 10, 1-63.

Strandburg-Peshkin Ariana, Papageorgiou Danai, Crofoot Margaret C., \& Farine Damien R. (2018). Inferring influence and leadership in moving animal groups. Philosophical Transactions of the Royal Society B: Biological Sciences, 373(1746), 20170006.

Street, S. E., Navarrete, A. F., Reader, S. M., \& Laland, K. N. (2017). Coevolution of cultural intelligence, extended life history, sociality, and brain size in primates. Proceedings of the National Academy of Sciences, 114(30), 7908-7914.

Sugiyama, L. S. (2004). Illness, injury, and disability among Shiwiar forager-horticulturalists: Implications of health-risk buffering for the evolution of human life history. American Journal of Physical Anthropology, 123(4), 371-389.

Tiger, L. (1970). Dominance in human societies. Annual Review of Ecology and Systematics, 1(1), 287-306.

Tiger, L. \& Fox, R. (1971). The imperial animal. Holt, Rinehart and Winston.

Tiger, L. \& Fox, R. (1997). The imperial animal, volume 4141. Transaction Publishers.

Todd, P. M., Hills, T. T., \& Robbins, T. W., Eds. (2012). Cognitive Search. The MIT Press.

Tokuyama, N. \& Furuichi, T. (2017). Leadership of old females in collective departures in wild bonobos (Pan paniscus) at Wamba. Behavioral Ecology and Sociobiology, 71(3), 55. 
Tooby, J. \& Cosmides, L. (1996). Friendship and the banker's paradox: Other pathways to the evolution of adaptations for altruism. Proceedings-British Academy, 88, 119-144.

Tooby, J., Cosmides, L., \& others (1996). Friendship and the banker's paradox: Other pathways to the evolution of adaptations for altruism. In Proceedings-British Academy, volume 88 (pp. 119-144).: Oxford University Press Inc.

Tooby, J., Cosmides, L., \& Price, M. E. (2006). Cognitive adaptations for n-person exchange: the evolutionary roots of organizational behavior. Managerial and Decision Economics, 27(2-3), 103-129.

Trapanese, C., Meunier, H., \& Masi, S. (2018). What, where and when: spatial foraging decisions in primates. Biological Reviews.

Trivers, R. L. (1974). Parent-offspring conflict. Integrative and Comparative Biology, 14(1), 249-264.

Underwood, B. \& Moore, B. (1982). Perspective-taking and altruism. Psychological Bulletin, 91(1), 143.

Van Vugt, M. (2006). Evolutionary origins of leadership and followership. Pers Soc Psychol Rev, 10(4), $354-71$.

Van Vugt, M. \& Kurzban, R. (2007). Cognitive and Social Adaptations for Leadership and Followership. Evolution and the social mind: Evolutionary psychology and social cognition, 9, 229.

Van Vugt, M. \& Tybur, J. M. (2014). The evolutionary foundations of hierarchy: Status, dominance, prestige, and leadership. In D. M. Buss (Ed.), Handbook of Evolutionary Psychology (pp. 788-809). Wiley, 2nd edition.

Vehrencamp, S. L. (1983). A model for the evolution of despotic versus egalitarian societies. Animal Behaviour, 31(3), 667-682.

von Rueden, C. \& Jaeggi, A. V. (2016). Mens status and reproductive success in 33 nonindustrial societies: Effects of subsistence, marriage system, and reproductive strategy. Proceedings of the National Academy of Sciences, (pp. 201606800).

von Rueden, C. R., Gurven, M., Kaplan, H., \& Stieglitz, J. (2014). Leadership in an Egalitarian Society. Hum Nat.

Voyer, D., Postma, A., Brake, B., \& Imperato-McGinley, J. (2007). Gender differences in object location memory: A meta-analysis. Psychonomic Bulletin \& Review, 14(1), 23-38.

Voyer, D. \& Voyer, S. D. (2014). Gender differences in scholastic achievement: A meta-analysis. Psychological Bulletin, 140(4), 1174.

Walker, R. S., Hill, K. R., Flinn, M. V., \& Ellsworth, R. M. (2011). Evolutionary history of hunter-gatherer marriage practices. PloS one, 6(4), e19066.

Webster, T. H. \& Wilson Sayres, M. A. (2016). Genomic signatures of sex-biased demography: progress and prospects. COGEDE Current Opinion in Genetics \& Development, 41, 62-71. OCLC: 6795086333.

Whiten, A. \& Byrne, R. W. (1997). Machiavellian Intelligence II: Extensions and Evaluations. Cambridge University Press. Google-Books-ID: bV9yeFV6_ckC.

Wiessner, P. (2002). Hunting, healing, and hxaro exchange A long-term perspective on !Kung (Ju/'hoansi) large-game hunting. Evolution and Human Behavior, 23(6), 407-436.

Wiessner, P. W. (2014). Embers of society: Firelight talk among the ju/'hoansi bushmen. Proceedings of the National Academy of Sciences, 111(39), 14027-14035.

Willems, E. P. \& van Schaik, C. P. (2017). The social organization of Homo ergaster: Inferences from anti-predator responses in extant primates. Journal of Human Evolution, 109, 11-21. 
Wilson, D. E. \& Reeder, D. M. (2005). Mammal species of the world: a taxonomic and geographic reference, volume 1. JHU Press.

Wilson, D. S., Near, D., \& Miller, R. R. (1996). Machiavellianism: a synthesis of the evolutionary and psychological literatures. Psychological Bulletin; Psychological Bulletin, 119(2), 285.

Woodburn, J. (1982). Egalitarian societies. Man, 17(3), 431-451.

Zeki, S. (2015). A massively asynchronous, parallel brain. Philosophical Transactions of the Royal Society B: Biological Sciences, 370(1668), 20140174.

Zeng, T. C., Aw, A. J., \& Feldman, M. W. (2018). Cultural hitchhiking and competition between patrilineal kin groups explain the post-Neolithic Y-chromosome bottleneck. Nature Communications, 9(1), 2077. 